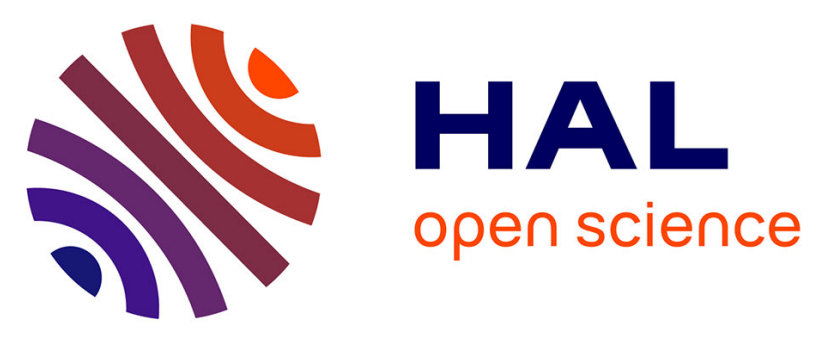

\title{
Seismic-induced damage detection through parallel force and parameter estimation using an improved interacting Particle-Kalman filter
}

Subhamoy Sen, Antoine Crinière, Laurent Mevel, Frédéric Cérou, Jean Dumoulin

\section{To cite this version:}

Subhamoy Sen, Antoine Crinière, Laurent Mevel, Frédéric Cérou, Jean Dumoulin. Seismic-induced damage detection through parallel force and parameter estimation using an improved interacting Particle-Kalman filter. Mechanical Systems and Signal Processing, 2018, 110, pp.231 - 247. 10.1016/j.ymssp.2018.03.016 . hal-01806896

\section{HAL Id: hal-01806896 https://hal.inria.fr/hal-01806896}

Submitted on 4 Jun 2018

HAL is a multi-disciplinary open access archive for the deposit and dissemination of scientific research documents, whether they are published or not. The documents may come from teaching and research institutions in France or abroad, or from public or private research centers.
L'archive ouverte pluridisciplinaire HAL, est destinée au dépôt et à la diffusion de documents scientifiques de niveau recherche, publiés ou non, émanant des établissements d'enseignement et de recherche français ou étrangers, des laboratoires publics ou privés. 


\title{
Seismic-induced damage detection through parallel force and parameter estimation using an improved interacting Particle-Kalman filter
}

\author{
Subhamoy Sen ${ }^{\mathrm{a}}$, Antoine Crinière ${ }^{\mathrm{b}, \mathrm{c}}$, Laurent Mevel $^{\mathrm{b}, \mathrm{c}, *}$, Frédéric Cérou ${ }^{\mathrm{d}}$, Jean Dumoulin ${ }^{\mathrm{c}, \mathrm{b}}$ \\ ${ }^{a}$ Indian Institute of Technology Mandi, HP, India \\ ${ }^{b}$ Inria, I4S, Campus de Beaulieu, 35042 Rennes, France \\ ${ }^{c}$ IFSTTAR, COSYS, SII, 44340 Bouguenay, Nantes, France \\ ${ }^{d}$ Inria, Aspi, Campus de Beaulieu, 35042 Rennes, France
}

\begin{abstract}
Standard filtering techniques for structural parameter estimation assume that the input force is either known or can be replicated using a known white Gaussian model. Unfortunately for structures subjected to seismic excitation, the input time history is unknown and also no previously known representative model is available. This invalidates the aforementioned idealization. To identify seismic induced damage in such structures using filtering techniques, force must therefore also be estimated. In this paper, the input force is considered to be an additional state that is estimated in parallel to the structural parameters. Two concurrent filters are employed for parameters and force respectively. For the parameters, an interacting Particle-Kalman filter is used to target systems with correlated noise. Alongside this, a second filter is used to estimate the seismic force acting on the structure. In the proposed algorithm, the parameters and the inputs are estimated as being conditional on each other, thus ensuring stability in the estimation. The proposed algorithm is numerically validated on a sixteen degrees-of-freedom mass-spring-damper system and a five-story building structure. The stability of the proposed filter is also tested by subjecting it to a sufficiently long measurement time history. The estimation results confirm the applicability of the proposed algorithm.

Keywords: Stochastic system identification, Kalman filtering, Particle filtering, Parameter tracking, Vibration monitoring, Seismic input, Force estimation
\end{abstract}

\section{Introduction}

Stochastic parameter estimation problems are characteristically categorized as nonlinear stochastic inverse problems. The related forward problem nonlinearly maps a set of model parameters to the corresponding measurements. Among the existing methods for inversely estimating the parameters, methods defined

${ }^{*}$ Corresponding author; Email address: laurent.mevel@inria.fr ; Phone number: +33240847325

Preprint submitted to Mechanical Systems and Signal Processing

June 4, 2018 
using a state space formulation are found to be popular [1-7] due to the relative ease in the estimation. Within this scope, filtering based recursive online system estimation techniques [8-10] are shown to be more efficient in using available measurements.

Filtering-based inverse estimation recursively employs (i) the Bayesian belief propagation technique (i.e. Chapman-Kolmogorov equation) to model the current estimate evolving over time and (ii) subsequent minimum mean square error (MMSE) based correction of the current estimate using the available measurements.

To execute the Chapman-Kolmogorov equation in discrete time, a state space model is needed to replicate the system dynamics in discrete time. For filtering-based parameter estimation algorithms, the system is usually defined using a state-space formulation of the dynamic model with parameters additionally appended to the unobserved state vector and collectively observed through the response measurements. Due to the nonlinear relationship of the unobserved parameters (as augmented states) to their respective observation (i.e. measurements), parameter estimation problems are inherently nonlinear. Over the years, Particle Filter (PF) [11-13] has been established as a better estimator than several other nonlinear variants of Kalman filter (KF) (e.g. Extended [8] or Unscented [14, 15] KF) for nonlinear problems [16-18]. However, the relatively high computational cost of PF is sometimes the major concern [19].

In most of the filtering-based parameter estimation algorithms, the system states and parameters are usually estimated jointly as an extended state vector [16, 20-22]. Being model based, these filters optimally estimate the parameters of a quasi-steady model of the real dynamic system. Nonetheless, any time variance in the system dynamics may cause the estimation to completely diverge yielding a false or infeasible solution. By decoupling the estimation of states and parameters (also called Rao-Blackwellisation [23-25]) and applying separate but interacting filtering strategies that attempt conditional estimation of states based on parameters and vice versa, time varying system model parameters can be optimally estimated. The benefit of such dual estimation over joint estimation strategies is discussed in [26]. Along similar lines, an interacting filtering strategy has been employed in [27] for damage detection in a flexible plate, where a particle filter is employed on a reduced order numerical model followed by an update of the particle positions through an Extended Kalman Filter (EKF).

Techniques for dual estimation of states and parameters by coupling two concurrent EKFs were proposed in [28, 29], whereas a dual estimation technique with two parallel PFs was used in [30, 31]. This approach not only ensures greater stability in the estimation, but also helps to keep the system dimension 
within limits. However, while the EKF based algorithms are reported to be not efficient with highly nonlinear systems [32], implementation of PF based dual estimation raises concerns regarding computational expense [19]. It should be noted that, while the parameter estimation is a nonlinear problem, the state estimation focuses on a linear process model. One can thus exploit this by engaging different filter types for state and parameters, such as Interacting Particle-Kalman filters (IPKF) [33] which make a clever use of costly PF for parameter estimation while standard KF handles the linear state estimation problem. Such techniques have a definite computational cost, which can be handled by integrating them in parallel computing methods (GPGPU) as in [34]. The major advantage of IPKF is that, with the strategy of decoupling the estimation of states and parameters, the state dimension can always be maintained at a reasonable size.

Ultimately, the optimal convergence of any filtering based parameter estimation algorithm depends on the information on the system input. Traditionally, while the time series of the system input is usually unknown, a stationary white Gaussian noise (WGN) model of it is however assumed to be available. Nevertheless, for systems subjected to non-stationary unknown input, traditional approaches fail to provide an optimal solution. A typical example would be the problem of monitoring structural health with seismic force as input which can not be modeled as a stationary WGN. A breakthrough can however be achieved by recursively updating the information about the seismic force and then supplying this information to the damage detection filter to achieve optimal structural health estimation.

In order to estimate the system input parallel to state, an unbiased minimum-variance linear state estimation filter without any a priori assumption on the input was pioneered in [35]. A practical method improving the filter used in [35] was later proposed in [36]. Other relevant works on joint state and input estimation involve the development of unbiased minimum variance optimal filters with no direct transmission term in the system formulation [37]. A modification is then proposed in [38] that relates a system to its direct transmission term. In [39], similar filters targeting issues related to numerical instability in the estimation, especially when the systems are redundantly instrumented, were proposed. An augmented KF (AKF) approach for input estimation was developed in [40]. However, he concluded that with AKF, the unobservability of the system may lead to instability in the estimation.

As mentioned above, estimating the force acting on a time invariant system using a dual PF approach was attempted in [31]. In a more recent study [41], joint input-parameter estimation was discussed for a time-varying system subjected to seismic excitation using the UKF technique. Their proposal is based on an AKF approach that augments the parameters and system inputs together in a very large state vector. 
Additionally, the system under consideration is time invariant with no sudden change in parameters in the course of estimation.

[42] discussed an EKF-based joint input-state-parameter estimation which handles all three components in an extended state vector. When implemented with particle filtering, this strategy may become intractable since a large dimensional state space has to be explored with particles that might be computationally impractical. [43] proposed a coupled DKF-UKF algorithm in which the DKF (Dual KF) algorithm estimates the input required for an UKF filter that estimates the state and parameters. Again, no sudden change in parameters (and therefore in the system) is addressed in this approach.

It is evident from the above discussion that, while there exists sufficient research on joint state and input identification for time invariant linear systems, similar research for time varying systems is scarce. Force estimation for a time varying system is however crucial, especially for typical SHM problems, since the anomalies in structural systems are mostly due to some rare events involving high magnitude forces for which no prior prediction can be made. Thus, in order to develop a robust SHM algorithm, these uncertain forces need to be estimated alongside and communicated to the core SHM algorithm for greater precision. In this paper, we develop a novel technique to detect damage in the presence of non-stationary input. A two-stage filtering strategy has been adopted, in which the first filter detects damage in the system while the second estimates the required force statistics in parallel. The required input information is estimated employing a KF parallel to the IPKF.

With seismic force as input, the innovation uncertainty of each KF is due to: i) inexact parameters, ii) error in the input, and, iii) measurement noise. Within each KF, this approach considers seismic force as WGN and assumes that the innovation is due to the inexact input only. This makes it easier to obtain a first estimate for the force by inverse mapping of the innovation. Obviously, this estimate is not conditional upon the current measurement and therefore is not optimal. The force filter then observes this predicted force estimate through measurements and error in the prediction is estimated. The prior estimate for force is then corrected to yield an estimate of the input that is conditional upon the current measurement. Updated information regarding the input is then fed back to the IPKF filter to update the covariance of the noise process.

Most filtering techniques assume that there is no correlation between the process noise and the measurement noise. However, the correlation becomes vital, especially for mechanical systems whose dynamics are defined using ambient forcing as the process noise (and not as system input), and the acceleration as 
measurement. Eventually, for such system descriptions, the process noise and the measurement noise are correlated. However, this invalidates the standard gain estimation that assumes that there is no correlation between the noise processes. For damage detection, the IPKF filter used in [33] has been modified to handle a system with correlated noise process. There is some literature that discusses similar correlated noise process in estimation e.g., [44-46]. The correlation has been taken into consideration in this paper and the derivation of the gain specific to this problem is presented in the Appendix.

Last, the performance of particle filter based estimation of high dimensional parameters is enhanced by defining a new evolution strategy for the particles. This strategy is based on the trend of the parameter evolution and discourages abrupt changes/updates to the parameters by avoiding infeasible estimation and subsequent divergence in the solution. For the numerical problem, a sixteen DOF system has been excited using El Centro earthquake (May 18, 1940 in CA, USA) vibration data (Data source: http://peer. berkeley.edu/research/motions/) and the proposed algorithm is employed to identify the damage online. A second numerical example is considered using a 3D lumped mass approximation of a base-excited five-story building. The robustness and stability of the proposed filter were further tested for a prolonged time and the results are found to be promising.

\section{Problem definition}

The differential equation governing the dynamics of a mechanical system with $(n \times n)$ order mass, damping and stiffness matrices being $\mathbf{M}, \mathbf{C}$ and $\mathbf{K}$ respectively subjected to seismic excitation $\ddot{\mathbf{q}}_{g}(t)$ can be represented as:

$$
\mathbf{M} \ddot{\mathbf{q}}(t)+\mathbf{C} \dot{\mathbf{q}}(t)+\mathbf{K q}(t)=-\mathbf{M} \tau \ddot{\mathbf{q}}_{g}(t)+\tilde{\mathbf{v}}(t)
$$

where $\mathbf{q}(t), \dot{\mathbf{q}}(t)$ and $\ddot{\mathbf{q}}(t)$ are $(n \times 1)$ order displacement, velocity and acceleration respectively. $\ddot{\mathbf{q}}_{g}(t)$ is the seismic excitation subjected to the structure and $\tilde{\mathbf{v}}(t)$ is the ambient forcing acting on the structure. $\tau$ is a matrix that defines how seismic excitation at some nodes affects the whole structure. Thus, to define this base excited system with a fixed-base, the actual base excitation has been replaced with equivalent inertia force at nodes, i.e. $-\mathbf{M} \tau \ddot{\mathbf{q}}_{g}(t)$. The simulated responses are therefore relative to the fixed base. The state space representation of the dynamics is presented below with the process and measurement noises where 
$(2 n \times 1)$ order state vector is observed by a $(m \times 1)$ output measurement collected at $m$ instrumented DOFs.

$$
\begin{aligned}
\dot{\mathbf{x}}(t) & =\mathbf{F}_{c} \mathbf{x}(t)+\mathbf{B}_{c} \mathbf{u}(t)+\mathbf{G}_{c} \mathbf{v}(t) \\
\mathbf{y}(t) & =\mathbf{H}_{c} \mathbf{x}(t)+\mathbf{D}_{c} \mathbf{u}(t)+\mathbf{L}_{c} \mathbf{v}(t)+\mathbf{w}(t)
\end{aligned}
$$

where $[\mathbf{u}(t)]_{n \times 1}=\tau \ddot{\mathbf{q}}_{g}(t) . \mathbf{F}_{c}, \mathbf{B}_{c}, \mathbf{H}_{c}$ and $\mathbf{D}_{c}$ are time dependent state, input, measurement and direct transmission matrices respectively defined in continuous time domain. Details of all the matrices along with their dimensions are given below:

$$
\begin{gathered}
\mathbf{x}(t)=\left[\begin{array}{ll}
\mathbf{q}(t)^{T} & \dot{\mathbf{q}}(t)^{T}
\end{array}\right]_{2 n \times 1}^{T} \quad \text { and } \quad \mathbf{F}_{c}=\left[\begin{array}{cc}
0 & \mathbf{I} \\
-\mathbf{M}^{-1} \mathbf{K} & -\mathbf{M}^{-1} \mathbf{C}
\end{array}\right]_{2 n \times 2 n} ; \\
\left.\mathbf{B}_{c}=\left[\begin{array}{c}
0 \\
-\mathbf{I}
\end{array}\right]_{2 n \times n}, \mathbf{G}_{c}=\left[\begin{array}{c}
0 \\
\mathbf{M}^{-1}
\end{array}\right]_{2 n \times n}, \quad \text { and } \quad \mathbf{H}_{c}=\left[\begin{array}{ll}
\mathbf{l}_{m}\left[\mathbf{M}^{-1} \mathbf{K}\right. & -\mathbf{M}^{-1} \mathbf{C}
\end{array}\right]\right]_{m \times 2 n}, \\
\mathbf{D}_{c}=\left[\begin{array}{ll}
\left.-\mathbf{l}_{m} \mathbf{I}\right]_{m \times n}, \quad \text { and } & \mathbf{L}_{c}=\left[\mathbf{l}_{m} \mathbf{M}^{-1}\right]_{m \times 2 n}
\end{array}\right.
\end{gathered}
$$

$[\mathbf{v}(t)]_{n \times 1}$ is the process noise for the system that takes care of the model uncertainty $\left(\mathbf{v}_{m}(t)\right)$ and the ambient forcing $(\tilde{\mathbf{v}}(t))$. Thus $\mathbf{v}(t)$ can be considered as $\mathbf{v}(t)=\mathbf{v}_{m}(t)+\tilde{\mathbf{v}}(t)$. $\mathbf{w}(t)$ is the sensor noise in the measurement. $\mathbf{l}_{m}$ is an $m \times n$ matrix that is defined based on the sensor locations and measured DOFs in order to choose the response at the measured DOFs only.

Since discrete measurements will be used for the estimation, the discrete time formulation of Equation 2 can be presented with $\mathbf{x}_{k}, \mathbf{y}_{k}, \mathbf{F}, \mathbf{B}, \mathbf{G}, \mathbf{H}, \mathbf{D}, \mathbf{L}, \mathbf{u}_{k}, \mathbf{v}_{k}$ and $\mathbf{w}_{k}$ as the discrete time counterparts against their corresponding continuous time entities. The system model is defined as time varying since the stiffness parameters vary in time. The corresponding state matrix $\mathbf{F}$ and measurement matrix $\mathbf{H}$ are defined with their dependence on the stiffness parameters $\theta$. Mass parameters are however considered to be time invariant and known a priori. Accordingly, the mass matrix and its dependants, i.e. B, G, D and $\mathbf{L}$ are assumed to be constant and known from now on.

$$
\begin{aligned}
\mathbf{x}_{k} & =\mathbf{F}_{k} \mathbf{x}_{k-1}+\mathbf{B} \mathbf{u}_{k}+\mathbf{G v}_{k} \\
\mathbf{y}_{k} & =\mathbf{H}_{k} \mathbf{x}_{k}+\left\{\mathbf{D} \mathbf{u}_{k}+\mathbf{L} \mathbf{v}_{k}+\mathbf{w}_{k}\right\}
\end{aligned}
$$

where $\mathbf{F}_{k}=\mathbf{F}\left(\theta_{k}\right)$ and $\mathbf{H}_{k}=\mathbf{H}\left(\theta_{k}\right)$.

Here, the discrete time input matrix $\mathbf{u}_{k}$ signifies the input to which the system is subjected after $(k-$ $1)^{t h}$ time instant, which drove the system states $\mathbf{x}_{k-1}$ to $\mathbf{x}_{k}$. The system states are further observed at 
the $k^{\text {th }}$ time instant as measurement $\mathbf{y}_{k}$. System state matrix $\mathbf{F}$ and measurement matrix $\mathbf{H}$ are assumed here to be functions of parameter $\theta_{k}$, which denotes the parameter values during the time interval $k-1^{t h}$ to $k^{\text {th }}$ time instant. Process uncertainty at $k^{\text {th }}$ instant is replicated using a realization of process noise $\mathbf{v}_{k}$ drawn from a Gaussian distribution of zero mean and time invariant covariance $\mathbf{Q}$. The measurement $\mathbf{y}_{k}$ is finally contaminated with measurement noise at $k^{\text {th }}$ instant. The time indices for the stochastic variables are selected as in [47].

The problem assumes that the stochastic properties of both process noise $\mathbf{v}_{k}$ and sensor noise $\mathbf{w}_{k}$ are known and therefore can be modeled as WGN with constant covariance $\mathbf{Q}$ and $\mathbf{R}$ respectively. The possibility of any mutual correlation between $\mathbf{u}_{k}, \mathbf{v}_{k}$ and $\mathbf{w}_{k}$ is ignored. Last, a WGN model is needed for the stochastic input $\mathbf{u}_{k}$, which is basically the seismic force with no previously known available model. The basic objective of this proposal is therefore to estimate the unknown and non-stationary input alongside states and parameters and then feed this information back for optimal parameter estimation.

In this context, a crucial aspect should be noted. Measurement uncertainty (see bracketed terms in Equation $3 b$ ) is not only due to sensor noise $\mathbf{w}_{k}$, but also to the stochastic nature of $\mathbf{v}_{k}$ and $\mathbf{u}_{k}$. Obviously, as a combination of $\mathbf{v}_{k}, \mathbf{u}_{k}$ and $\mathbf{w}_{k}$, the resulting measurement noise is thus correlated to $\mathbf{v}_{k}$ and $\mathbf{u}_{k}$, even if the sensor noise $\mathbf{w}_{k}$ is uncorrelated to any of the other noises. Nevertheless, standard filtering gain, which is based on an assumption that process and measurement noises are orthogonal, is no longer valid.

In this paper, the existing IPKF algorithm [33] is used as the basis for detecting damage in the system in the presence of measurement noise. However, since IPKF employs a traditional KF that does not take correlation between the noises into account, an improvement in the gain estimation is made to tackle the correlated noise processes in the system (see Appendix for more details). The capabilities of the IPKF strategy used are further enhanced by developing an additional force filter to make it robust against nonstationary input force statistics.

\section{The present approach}

The IPKF algorithm, developed in [33] and further improved for practical implementation in [34], is an efficient approach for handling parameter estimation for time varying systems. The idea is to nest a set of linear KFs for state estimation within a PF environment that estimates the parameters. This makes it possible to use relatively less expensive linear KF for linear state estimation problem while the costly PF approach is employed only for parameter estimation. In turn, this approach not only helps lower computational costs by 
reducing the dimension of the state vector required to be estimated by particles, but it also ensures stability in the estimation for time varying systems by decoupling the estimation of states and parameters.

Bayesian belief propagation requires that an explicit analytical integration be solved for the entire domain of states. This is however straight-forward as long as the problem is linear and the states are assumed to be Gaussian. The current problem is nonlinear, so explicit analytical integration over the entire parameter space is not possible. Furthermore, no assumptions regarding the stochastic nature of the parameter states are meant to be enforced. PF attempts a particle approximation of this integration by propagating the system uncertainty through a cloud of $N$ independent parameter particles $\Xi_{k}=\left[\xi^{1}, \xi^{2}, \cdots, \xi^{N}\right]$. The system dynamics are defined by the evolution of this particle set over time. At any arbitrary time step $k$, the evolution of an arbitrary particle $\xi_{k-1}^{i}$ is basically a random perturbation around its current position:

$$
\xi_{k}^{i}=\xi_{k-1}^{i}+\mathbb{N}\left(\delta \xi_{k} ; \sigma_{k}^{\xi}\right)
$$

where a Gaussian blurring is performed on $\xi_{k-1}^{i}$ with a shift $\delta \xi_{k}$ and a spread of $\sigma_{k}^{\xi}$. The evolved particles are then weighted, based on their likelihood against the current measurement.

\subsection{The IPKF algorithm}

In the IPKF, state estimation is performed using a set of KFs within the PF environment where each of the KFs is associated with one value of the corresponding parameter particles, $\xi_{k}^{i} \in\left\{\xi_{k}^{i}\right\}$, for which the state estimation is performed. The $\left\{\xi_{k}^{i}\right\}$ set is generated around the old particle set $\left\{\xi_{k-1}^{i}\right\}$ by perturbation. The KF is thus employed to estimate the states, $\mathbf{x}_{k}$, while the PF does a particle approximation (weighted averaging) on all particles in $\left\{\xi_{k}^{i}\right\}$ to yield an estimate for the parameters, $\theta_{k}$.

Each of the evolved parameter particles is used to follow the system matrices as $\mathbf{F}_{k}^{i}=\mathbf{F}\left(\xi_{k}^{i}\right)$ and $\mathbf{H}_{k}^{i}=$ $\mathbf{H}\left(\xi_{k}^{i}\right)$. The stochastic nature of the system input due to ambient and seismic forces is defined using a stationary WGN model for $\mathbf{v}_{k}$ with covariance $\mathbf{Q}$ and a non-stationary WGN model of covariance $\mathbf{S}_{k}$ for $\mathbf{u}_{k}$. The cumulative covariance for the process noise is therefore:

$$
\mathbb{Q}_{k}=\mathbf{B S}_{k} \mathbf{B}^{T}+\mathbf{G Q G}
$$

With this system model, KF estimates the system state conditioned on the current estimate of the parameter particle $\xi_{k}^{i}$. The state and measurement equations for the system presented in Equation 3 can thus be defined

\footnotetext{
${ }^{1} A+B \mathbb{N}(\mu ; \sigma)$ means $A+B z$ where $z$ follows $\mathbb{N}(\mu ; \sigma)$
} 
as:

$$
\begin{aligned}
& \mathbf{x}_{k}=\mathbf{F}_{k} \mathbf{x}_{k-1}+\mathbb{N}\left(0, \mathbb{Q}_{k}\right) \\
& \mathbf{y}_{k}=\mathbf{H}_{k} \mathbf{x}_{k}+\mathbb{N}\left(0, \mathbb{R}_{k}\right)
\end{aligned}
$$

where $\mathbb{R}_{k}=\mathbf{D S}_{k} \mathbf{D}^{T}+\mathbf{L Q} \mathbf{L}^{T}+\mathbf{R}$.

In what follows, the estimation procedure for this state-space system is described for an arbitrary time step $k$. The estimation involves propagating the current state estimate $\mathbf{x}_{k-1 \mid k-1}^{i}{ }^{2}$, through the system conditional on the current parameter estimate for each $i^{\text {th }}$ particle in order to predict $\mathbf{x}_{k \mid k-1}^{i}$. Predicted state estimate is then improved using current measurement $\mathbf{y}_{k}$ to obtain the posterior estimate $\mathbf{x}_{k \mid k}^{i}$. This process is repeated for all particles yielding a set of posterior estimates for states $\left\{\mathbf{x}_{k \mid k}^{i} ; \quad\right.$ for all $\left.i=1,2, \cdots, N\right\}$.

The prediction and correction steps of the KF for an arbitrary $i^{\text {th }}$ particle at $k^{\text {th }}$ time step are described as follows.

\section{Prediction}

$$
\begin{aligned}
\mathbf{x}_{k \mid k-1}^{i} & =\mathbf{F}_{k}^{i} \mathbf{x}_{k-1 \mid k-1}^{i} \\
\mathbf{P}_{k \mid k-1}^{i} & =\mathbf{F}_{k}^{i} \mathbf{P}_{k-1 \mid k-1}^{i} \mathbf{F}_{k}^{i T}+\mathbb{Q}_{k}
\end{aligned}
$$

Innovation

$$
\varepsilon_{k}^{i}=\mathbf{y}_{k}-\mathbf{H}_{k}^{i} \mathbf{x}_{k \mid k-1}^{i}
$$

\section{Correction}

$$
\begin{aligned}
\mathbf{x}_{k \mid k}^{i} & =\mathbf{x}_{k \mid k-1}^{i}+\mathbf{K}_{k}^{i} \varepsilon_{k}^{i} \\
\mathbf{P}_{k \mid k}^{i} & =\left(\mathbf{I}-\mathbf{K}_{k}^{i} \mathbf{H}_{k}^{i}\right) \mathbf{P}_{k \mid k-1}^{i}
\end{aligned}
$$

where $\mathbf{K}_{k}^{i}$ is the gain matrix. It can be verified from Equations $6 \mathrm{a}$ and $6 \mathrm{~b}$, that both the process and measurement noises (i.e. $\mathbb{N}\left(0, \mathbb{Q}_{k}\right)$ and $\mathbb{N}\left(0, \mathbb{R}_{k}\right)$ ) of the system are functions of $\mathbf{Q}$ and therefore obviously correlated. This is usual for the state space formulations of mechanical systems with acceleration response considered as measurement. Typical Kalman gain derivation, which assumes that there is no correlation between process and measurement noises, is thus not valid for this kind of formulation. The derivation of this gain matrix specific to this problem is discussed in the Appendix to this paper.

\footnotetext{
${ }^{2} \mathbf{x}_{i \mid j}$ represents the estimate of the random variable $\mathbf{x}$ at the $i^{t h}$ time instant providing the measurement including and up to time instant $j$.
} 


\subsection{Force filter}

Evidently, IPKF represents input force using a non-stationary WGN model with time varying covariance $\mathbf{S}_{k}$ (see Equation 5). Therefore, to achieve greater precision in parameter estimates, covariance of time varying input force has to be estimated recursively. Targeting this, the current proposal implements a second filter to estimate the time history of the system input $\mathbf{u}_{k}$ from which the input covariance $\mathbf{S}_{k}$ is estimated online $^{3}$

The force filter estimates the input force $\mathbf{u}_{k}$ as system state conditioned on the current parameter estimate, $\xi_{k}^{i}$. The system dynamics described in Equation $6 \mathrm{a}$ is therefore redefined separating out the input forces from the WGN model $\mathbb{N}\left(0, \mathbb{Q}_{k}\right)$ (see Equation 6a).

$$
\mathbf{x}_{k}^{(I)}=\mathbf{F}_{k} \mathbf{x}_{k-1}+\mathbf{B} \mathbf{u}_{k}+\mathbb{N}\left(0, \mathbf{G Q G}^{T}\right)
$$

where $\mathbf{x}_{k}^{(I)}$ is the modified state with the superscript $(I)$ signifying that the input information has been presented separately. This definition will help in identifying the relation between input force and output measurement which will be used further to define a system with input as system state. The system is further observed through the measurement $\mathbf{y}_{k}$ (see Equations $3 \mathrm{a}$ and $3 \mathrm{~b}$ ) as:

$$
\mathbf{y}_{k}=\mathbf{H}_{k} \mathbf{x}_{k}^{(I)}+\mathbf{D} \mathbf{u}_{k}+\mathbb{N}\left(0,\left\{\mathbf{L Q} \mathbf{L}^{T}+\mathbf{R}\right\}\right)
$$

Combining Equations 8 and 9, the measurement equation can be obtained as:

$$
\mathbf{y}_{k}=\mathbf{H}_{k} \mathbf{F}_{k} \mathbf{x}_{k-1}+\left(\mathbf{H}_{k} \mathbf{B}+\mathbf{D}\right) \mathbf{u}_{k}+\mathbb{N}\left(0,\left\{\left(\mathbf{H}_{k} \mathbf{G}+\mathbf{L}\right) \mathbf{Q}\left(\mathbf{H}_{k} \mathbf{G}+\mathbf{L}\right)^{T}+\mathbf{R}\right\}\right)
$$

It should be understood that the system presented in Equations 8-10 and Equation 6 actually represents the same system dynamics. However, when the system is estimated using Equations 7a-7e, the Kalman innovation (see Equation 7c) will be centered around $\left(\mathbf{H}_{k} \mathbf{B}+\mathbf{D}\right) \mathbf{u}_{k}$ with an associated uncertainty defined in Equation 10. The innovation, therefore, contains the information on the current force. Clearly, if an optimal estimation for the force is available, the uncertainty in the innovation estimation can be reduced.

In what follows, the estimation procedure for the system defined in Equation 8 with $\mathbf{u}_{k}$ as system state will be discussed. For each nested KF, current estimates (not the true value) of parameter and state affect the uncertainty in the innovation. From Equation 3b, it can be seen that the innovation uncertainty is dependent

\footnotetext{
${ }^{3} \mathbf{S}_{k}$ is calculated using particle approximation of force estimates for all particles and is described in detail together with other particle approximated entities in the next section.
} 
on: (i) inaccuracy in parameter estimates, (ii) measurement uncertainty and (iii) error in force estimates. We, however, force an assumption that the Kalman innovation $\varepsilon_{k}^{i}$ (see Equation 7c) for each particle is solely due to the input. This allows us to redefine innovation uncertainty (see Equation 7c) with its dependency on input force estimates as:

$$
\varepsilon_{k}^{i}=\mathbf{y}_{k}-\mathbf{H}_{k}^{i} \mathbf{x}_{k \mid k-1}^{i}=\left(\mathbf{H}_{k}^{i} \mathbf{B}+\mathbf{D}\right) \mathbf{u}_{k}^{i}+\mathbb{N}\left(0,\left\{\left(\mathbf{H}_{k}^{i} \mathbf{G}+\mathbf{L}\right) \mathbf{Q}\left(\mathbf{H}_{k}^{i} \mathbf{G}+\mathbf{L}\right)^{T}+\mathbf{R}\right\}\right)
$$

Here $\mathbf{u}_{k}^{i}$ is an estimate of seismic force $\mathbf{u}_{k}$ at $k^{t h}$ instant with an associated Gaussian error model.

By means of inverse mapping, the force can be defined as the combination of the normalized innovation $\varepsilon_{k}^{i}$ and a Gaussian error.

$$
\mathbf{u}_{k}^{i}=\left(\mathbf{H}_{k}^{i} \mathbf{B}+\mathbf{D}\right)^{\dagger} \mathcal{\varepsilon}_{k}^{i}-\left(\mathbf{H}_{k}^{i} \mathbf{B}+\mathbf{D}\right)^{\dagger}\left\{\mathbb{N}\left(0,\left(\mathbf{H}_{k}^{i} \mathbf{G}+\mathbf{L}\right) \mathbf{Q}\left(\mathbf{H}_{k}^{i} \mathbf{G}+\mathbf{L}\right)^{T}+\mathbf{R}\right)\right\}
$$

where $\uparrow$ signifies pseudo-inverse. An approximated process model for force estimation at any arbitrary time step $k$ thus can be defined as:

$$
\mathbf{u}_{k}^{i}=\hat{\mathbf{u}}_{k}^{i}+\mathbb{N}\left(0, Q_{k}^{i}\right)
$$

with $\hat{\mathbf{u}}_{k}^{i}=\left(\mathbf{H}_{k}^{i} \mathbf{B}+\mathbf{D}\right)^{\dagger} \varepsilon_{k}^{i}$ and $Q_{k}^{i}$ being:

$$
Q_{k}^{i}=\left(\mathbf{H}_{k}^{i} \mathbf{B}+\mathbf{D}\right)^{\dagger}\left\{\left(\mathbf{H}_{k}^{i} \mathbf{G}+\mathbf{L}\right) \mathbf{Q}\left(\mathbf{H}_{k}^{i} \mathbf{G}+\mathbf{L}\right)^{T}+\mathbf{R}\right\}\left(\mathbf{H}_{k}^{i} \mathbf{B}+\mathbf{D}\right)^{\dagger}
$$

Here, it can be observed that the prediction related to force depends on the particle estimates and thus has an index $i$ associated with it. This in turn demonstrates that force estimates are conditional on current state and parameter estimates. However, this prior estimate is conditional on the propagated system state $\mathbf{x}_{k \mid k-1}^{i}$ and therefore needs to be corrected.

Two different uncertainties are associated with the estimation of $\mathbf{u}_{k \mid k-1}^{i}$ : (i) external uncertainty with covariance $Q_{k}^{i}$ (due to process and measurement noise in the main system), (ii) internal uncertainty with covariance $\left(\mathbf{P}_{\mathbf{u}}\right)_{k \mid k-1}^{i}$ (due to estimation inaccuracies). The prediction for the mean $\left(\mathbf{u}_{k \mid k-1}^{i}\right)$ and covariance $\left(\left(\mathbf{P}_{\mathbf{u}}\right)_{k \mid k-1}^{i}\right)$ of the force can be obtained as:

$$
\begin{aligned}
\mathbf{u}_{k \mid k-1}^{i} & =\hat{\mathbf{u}}_{k}^{i} \\
\left(\mathbf{P}_{\mathbf{u}}\right)_{k \mid k-1}^{i} & =\operatorname{COV}\left[\mathbf{u}_{k}^{i} \mid \mathbf{y}_{1: k-1}\right]=\left(\mathbf{P}_{\mathbf{u}}\right)_{k-1 \mid k-1}^{i}+Q_{k}^{i}
\end{aligned}
$$

Here, the notation $\operatorname{COV}[\mathbf{x}]$ is analogous to $\operatorname{COV}[\mathbf{x}, \mathbf{x}]$, while later in this paper, the notation $\operatorname{COV}[\mathbf{x}, \mathbf{y}]$ is used to define covariance between two different variables $\mathbf{x}$ and $\mathbf{y}$. 
Predicted force estimation $\mathbf{u}_{k \mid k-1}^{i}$ must then be observed through the measurement to calculate the estimation error. From the measurement equation, the innovation associated with $\mathbf{u}_{k \mid k-1}^{i}$ can be estimated as:

$$
e_{k}^{i}=\mathbf{y}_{k}-\mathbf{H}_{k}^{i} \mathbf{x}_{k \mid k}^{i}-\left(\mathbf{H}_{k}^{i} \mathbf{B}+\mathbf{D}\right) \mathbf{u}_{k \mid k-1}^{i}
$$

Thus $\mathbf{u}_{k}$ acts as the unobserved state variable for the force filter, while $\mathbf{y}_{k}$ is the corresponding observation. The prior force estimates are then updated incorporating the correction though the force filter as:

$$
\mathbf{u}_{k \mid k}^{i}=\mathbf{u}_{k \mid k-1}^{i}+\left(\mathbf{K}_{\mathbf{f}}\right)_{k}^{i} e_{k}^{i} \quad \text { and } \quad\left(\mathbf{P}_{\mathbf{u}}\right)_{k \mid k}^{i}=\left[\mathbf{I}-\left(\mathbf{K}_{\mathbf{f}}\right)_{k}^{i}\left(\mathbf{H}_{k}^{i} \mathbf{B}+\mathbf{D}\right)\right]\left(\mathbf{P}_{\mathbf{u}}\right)_{k \mid k-1}^{i}
$$

where $\left(\mathbf{K}_{\mathbf{f}}\right)_{k}^{i}$ is the gain for the $i^{t h}$ force filter at $k^{\text {th }}$ time step which is obtained as:

$$
\left(\mathbf{K}_{\mathbf{f}}\right)_{k}^{i}=\operatorname{COV}\left[\mathbf{u}_{k}^{i} ; \mathbf{y}_{k} \mid \mathbf{y}_{1: k-1}\right] \operatorname{COV}\left[\mathbf{y}_{k} ; \mathbf{y}_{k} \mid \mathbf{y}_{1: k-1}\right]^{-1}=\left[\left(\mathbf{P}_{\mathbf{u}}\right)_{k \mid k-1}^{i} \mathbf{H}_{\mathbf{k}}^{\mathbf{f}}\right]\left[\mathbf{H}_{\mathbf{k}}^{\mathbf{f}}\left(\mathbf{P}_{\mathbf{u}}\right)_{k \mid k-1}^{i}\left(\mathbf{H}_{\mathbf{k}}^{\mathbf{f}^{i}}\right)^{T}+\mathbf{H}_{k}^{i} \mathbf{P}_{k \mid k}^{i}\left(\mathbf{H}_{k}^{i}\right)^{T}+\mathbf{R}\right]^{-1}
$$

where $\mathbf{H}_{\mathbf{k}}^{\mathbf{f}^{i}}=\left(\mathbf{H}_{k}^{i} \mathbf{B}+\mathbf{D}\right)$ is the observation matrix for the force filter.

Finally, the initial state estimates obtained using the IPKF are corrected by incorporating the corrected input information obtained through the force filter.

$$
\mathbf{x}_{k \mid k}^{i}=\mathbf{x}_{k \mid k}^{i}+\mathbf{B} \mathbf{u}_{k \mid k}^{i}
$$

This corrected estimate is then used as the prior estimate for the next IPKF iteration.

\subsection{Particle approximation}

In the following, the likelihood of each particle is calculated based on the innovation mean and covariance of each KF. This likelihood information is used to update the normalized weight for each particle. The weight of $i^{\text {th }}$ particle is then estimated as:

$$
w\left(\xi_{k}^{i}\right)=\frac{w\left(\xi_{k-1}^{i}\right) P\left(\mathbf{y}_{k} \mid \xi_{k}^{i}\right)}{\sum_{j=1}^{N} w\left(\xi_{k-1}^{j}\right) P\left(\mathbf{y}_{k} \mid \xi_{k}^{j}\right)}
$$

The particle approximations for the parameter and state are then estimated as:

$$
\mathbf{x}_{k \mid k}=\sum_{i=1}^{N} w\left(\xi_{k}^{i}\right) \mathbf{x}_{k \mid k}^{i} \quad \text { and } \quad \bar{\xi}_{k}=\sum_{i=1}^{N} w\left(\xi_{k}^{i}\right) \xi_{k}^{i}
$$

A time varying covariance $\mathbf{S}_{k}$ is defined for the non-stationary input $\mathbf{u}_{k}$, and is estimated by selecting a window of the past estimated inputs. This is obviously a conditional estimate of the covariance of the input estimates $\mathbf{u}_{k \mid k}$ and will be represented as $\mathbf{S}_{k \mid k}$.

$$
\mathbf{S}_{k \mid k}=\frac{1}{\delta k} \sum_{j=0}^{\delta k}\left\{\mathbf{u}_{k-j \mid k-j}-\overline{\mathbf{u}}_{k \mid k}\right\}^{2}
$$


where $\delta k$ is the window size and $\overline{\mathbf{u}}_{k \mid k}$ is the mean of force estimates within that window. $\mathbf{u}_{k \mid k}$ are the particle approximations of all the force particles:

$$
\mathbf{u}_{k \mid k}=\sum_{i=1}^{N} w\left(\xi_{k}^{i}\right) \mathbf{u}_{k \mid k}^{i}
$$

\subsection{Evolution strategy for particles}

In PF, the particle evolution plays a crucial role in driving the current estimates towards the actual solution. This involves replacing particles with low likelihood with new particle realizations generated from the high probability zone. The particles are commonly perturbed around their current position by adding a pre-fixed variability. This strategy does not utilize the history of particle evolution over time. Thus, if an unrealistic estimation (due to measurement outliers or numerical instability in the computation) occurs, it might affect the estimation procedure. An erroneous set of particles can even drive the estimate far away from the actual parameter. Sometimes this divergence is so strong, that it can never return to its desired position, leading to complete divergence of the algorithm.

For the time invariant system, when the target solution is fixed, a prefixed variability can still be practical. However, with time varying systems, the target solution changes its position over time. Thus, only persistent updates in the particle position should be allowed. This persistent change can be evaluated by taking a weighted average of the particle history and its current estimate. This does, however, slow down the update, but also to some extent restricts any abnormal updating from disrupting the estimation procedure.

In this paper, a dual strategy is proposed for parameter evolution that uses the memory of its past evolution trend (i) to shift the current particle position and (ii) to define the spread of the particle perturbation. The standard kernel smoothing for parameters leads to an over dispersed approximation of posterior covariance which might cause loss of information in the long run [48]. A shrinkage rule was proposed in $[49,50]$ by introducing a monotonically decreasing function $h$ which asymptotically approaches to 0 as $N$ tends to infinity. A similar strategy has been used in this paper to correct the over-dispersion problem of the posterior variance that attempts to shift the current particles towards their respective particle mean as:

$$
\xi_{k}^{* i}=\alpha \xi_{k-1}^{i}+(1-\alpha) \bar{\xi}_{k-1}
$$

$\bar{\xi}_{k}$ represents the particle mean of all the parameter particles at time instant $k$ while $\xi^{* i}{ }_{k}$ represents the shifted position of the particle. Each particle is thus shifted from its previous position $\xi_{k-1}^{i}$ towards its particle mean $\bar{\xi}_{k-1} . \alpha$ is a tuning parameter that decides how much weight should be assigned to the past history of the evolution and should be decided based on the number of available particles $N$. While a low value confers 
more confidence on the past history and thus ensures stable and smooth convergence, it slows down the convergence. On the other hand, with a high value for $\alpha$, new estimates are given more priority. Although it yields rapid updating, it may sometimes lead to divergence as well. A value within the range 0.8 to 0.95 usually gives good results.

To add variability to the current particle position $\xi_{k}^{i}$, instead of adding a constant perturbation, it is decided to exploit the evolution history for a proper selection of the perturbation magnitude. This ensures that if the parameter is changing, the variability magnitude will gradually increase, allowing the change to be adapted by widely-separated particles. On the other hand, if the parameter maintains an almost constant position, the variability slowly decreases and stops at a minimum fixed value $\sigma_{0}^{\xi}$, making it possible for the solution to be narrowed down and converge smoothly. This strategy is described below:

$$
\begin{aligned}
\Delta \xi_{k} & =\frac{1}{\bar{\xi}_{k-1}}\left|\left\{\bar{\xi}_{k-\delta k}-\bar{\xi}_{k-1}\right\}\right| \\
\sigma_{k}^{\xi} & =\left(1+\Delta \xi_{k}\right) \sigma_{0}^{\xi}
\end{aligned}
$$

Here $\delta k$ is the window length within which changes in parameter particles $\bar{\xi}_{k}$ are tracked. $|\bullet|$ is the modulus operation. Finally, the new particles for the next iteration are generated as:

$$
\xi_{k}^{i}=\xi_{k}^{* i}+\mathbb{N}\left(0, \sigma_{k}^{\xi}\right)
$$

The proposed approach is presented in the form of a pseudo-code in Algorithm 1.

\section{Numerical Validation}

Numerical studies are used to establish the applicability of this algorithm. Two test structures are considered (i) a 16 DOF mass-spring-dashpot system with a seismic excitation in one direction, and (ii) a five-story asymmetric building subjected to bi-directional seismic excitation. Details of each numerical simulation are presented below.

\subsection{DOF mass-spring-dashpot}

A sixteen degrees of freedom mass-spring-damper system is considered here as the test structure. The system is excited with El Centro earthquake excitation (May 18, 1940 in CA, USA, direction North-South) vibration data (Data source: http://peer.berkeley.edu/research/motions/). For the sake of practicality, it has been assumed that the earthquake arrived two seconds after the monitoring began and that damage is initiated at the third second of the procedure. All the mass blocks are assumed to have the same 


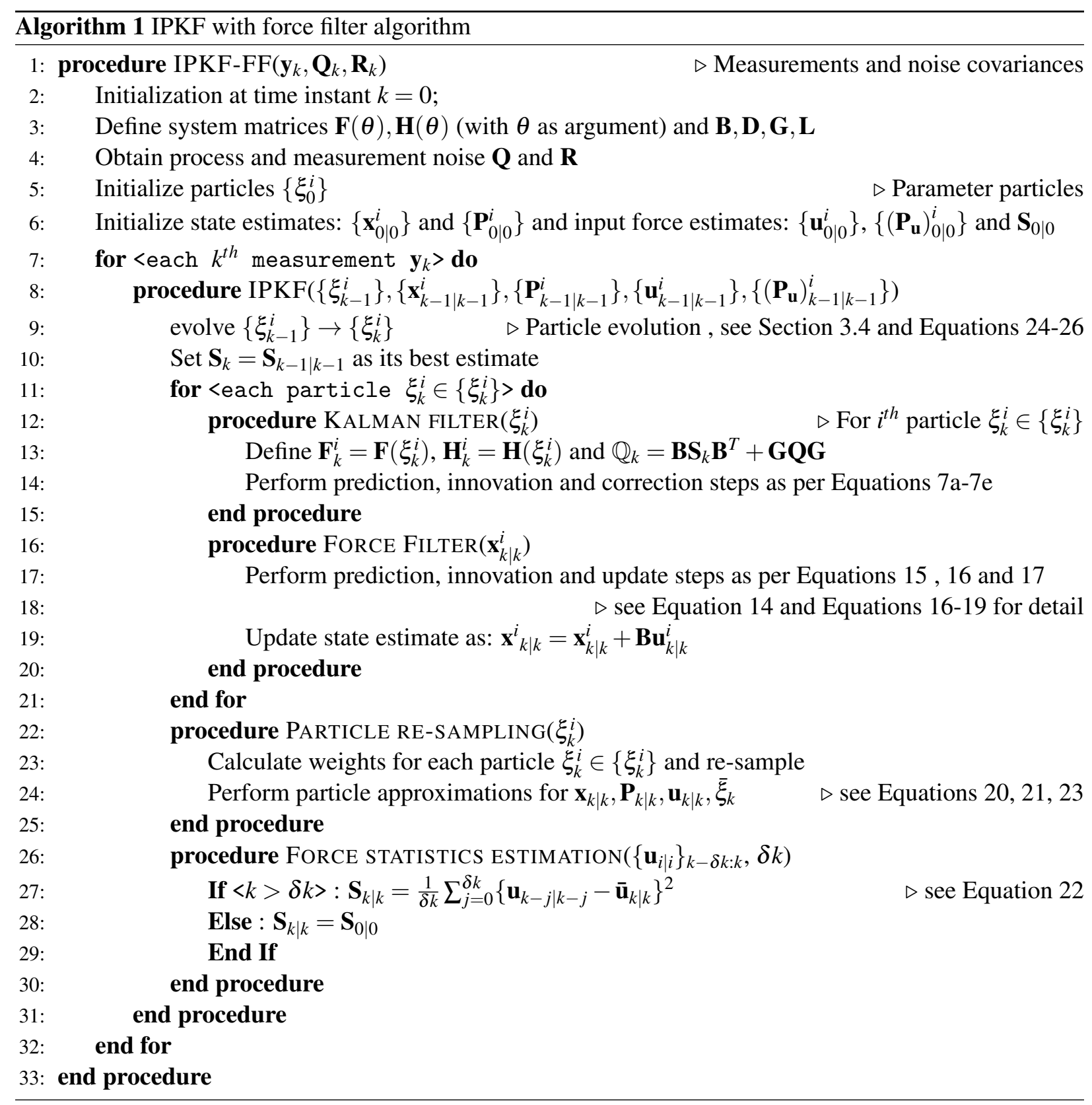


properties, with mass $10 \mathrm{~kg}$ and that the stiffness of all springs are $8,000 \mathrm{~N} / \mathrm{m}$. The schematic diagram is given in Figure 1. Damage is initiated at the sixth DOF by reducing spring stiffness to 2,000 $/ \mathrm{m}$.

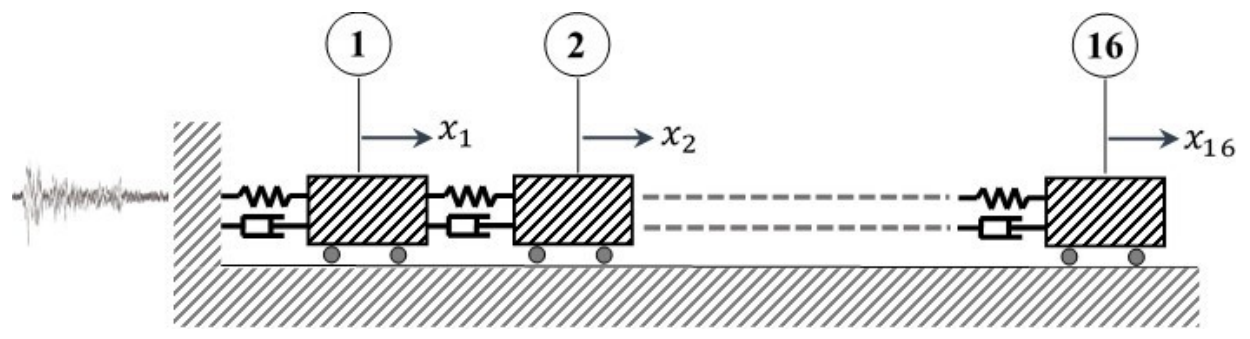

Figure 1: Schematic diagram of the 16 DOF test structure

The test structure is subjected to the seismic excitation at the first node. Apart from seismic forcing, a stationary WGN of variance 1 is applied as ambient excitation acting on all the nodes of the structure. Acceleration response is collected as measurement. Four different case studies are performed assuming 16, 8,4 and 2 available measurement channels. The locations of the instrumented nodes are selected as per this structure: $\left[16 / N_{m}: 16 / N_{m}: 16\right]$, where $N_{m}$ denotes the number of instrumented channels. The measurements are sampled at a frequency of $50 \mathrm{~Hz}$ for a signal length of 2048 samples. These measurements are contaminated with a stationary WGN of variance 0.1 . The proposed approach is then applied to the contaminated signal to simultaneously estimate state, parameter (i.e. indications of damage) and the input seismic excitation.

In practice, a good implementation of PF with an accurate model usually runs well with a few thousand particles [51]. For damage detection problems, in which the model is not accurate, the particle size may be slightly larger. Obviously the precision of the IPKF filter is proportional to the number of particles used. However, the cost of computation increases with the number of particles. Based on our previous experience with IPKF filter [33], this current proposal is simulated using 2,000 particles for which the precision is observed to be sufficient. Initial distribution for all the parameters is set to be Gaussian distributed with their mean set at their undamaged stiffness value with a coefficient of variation selected as $5 \%$. The parameters evolve over time based on their likelihood and no assumption regarding their distribution is applied.

A value of $10^{4} N^{2} / \mathrm{m}^{2}$ has been used as $\sigma_{0}^{\xi}$ while $\alpha$ has been selected as 0.95 for all case studies. For the first 100 iterations, a constant value for $\mathbf{S}_{k \mid k}$ of $100 \mathrm{~m}^{2} / \mathrm{s}^{4}$ has been supplied. The window lengths for tracking the force covariance (see Equation 22) and for tracking change in particles (see Equation 25) have been selected as 100 and 50 respectively. Zero order hold technique is employed for time discretization. 


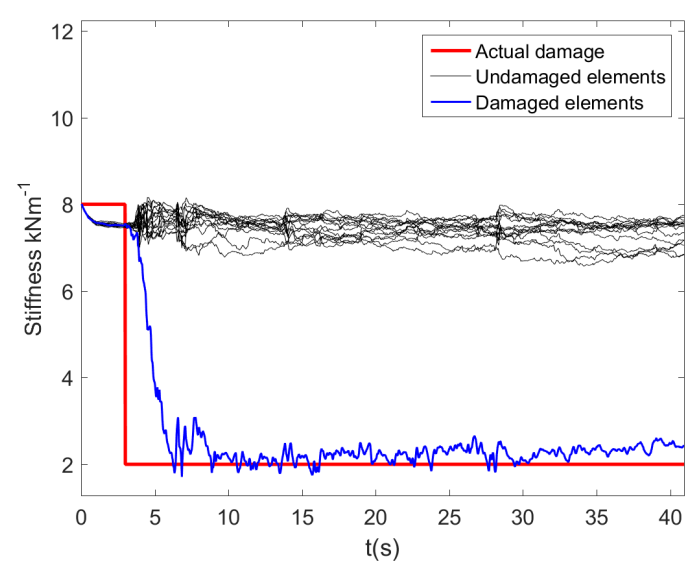

(a) 16 Channel measured signal

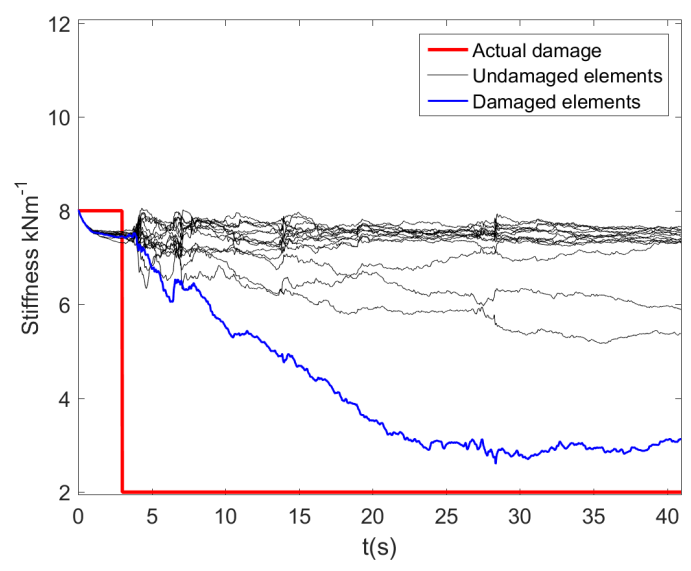

(c) 4 Channel measured signal

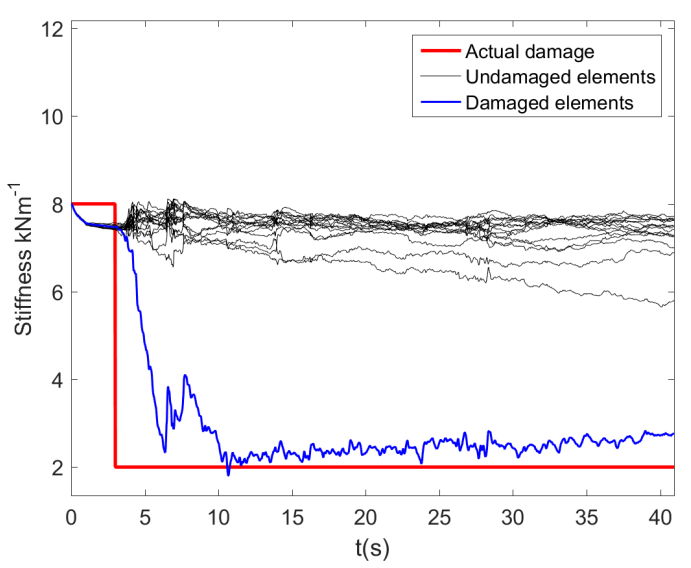

(b) 8 Channel measured signal

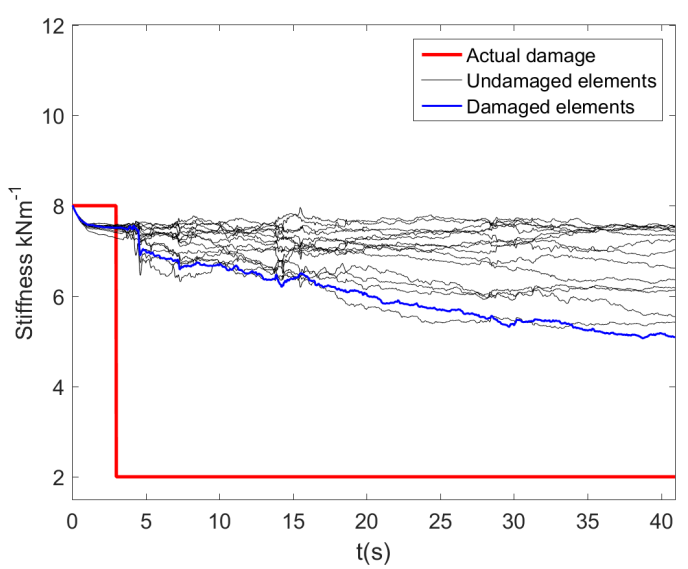

(d) 2 Channel measured signal

Figure 2: Parameter estimation for 16, 8, 4 and 2 channel measured signal 
The results of the parameter estimation presented in Figure 2 and Figure 3 describe the estimation of the forces compared against their true values. From Figures 2 and 3, it is evident that by reducing the number of available sensors, the quality of the estimation decreases. For a two channel measured signal, the damage has not been identified properly and the force estimation performance is also poor. However, it can be concluded that for a 16 DOF system, a minimum of 4 sensors can be used to estimate the damage in the system, while precisely estimating the input excitation. It can also be seen, that the damage estimation is quite prompt and sufficiently accurate to raise an alarm as soon as the damage occurs. Furthermore, we did not experience any occurrence of false alarm during this study.

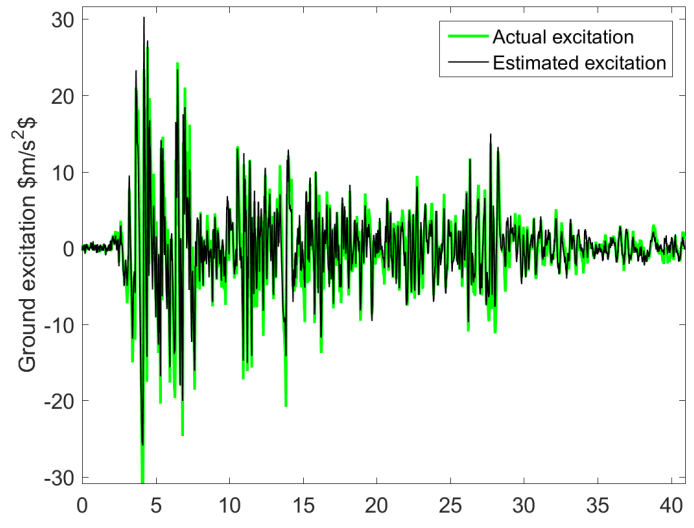

(a) 16 Channel measured signal

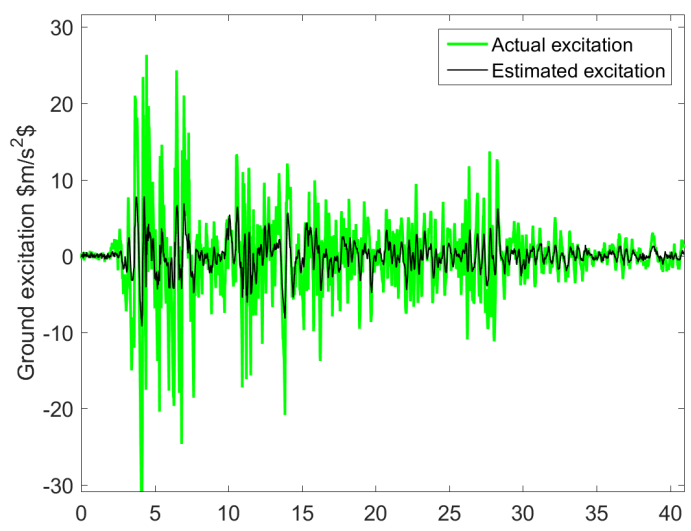

(c) 4 Channel measured signal

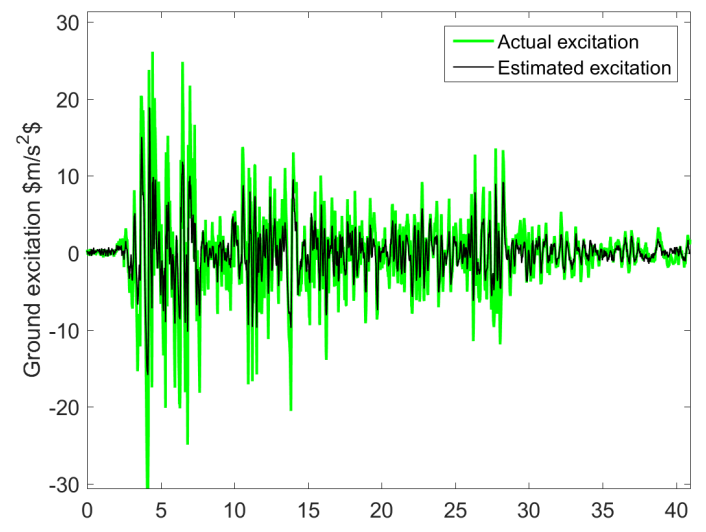

(b) 8 Channel measured signal

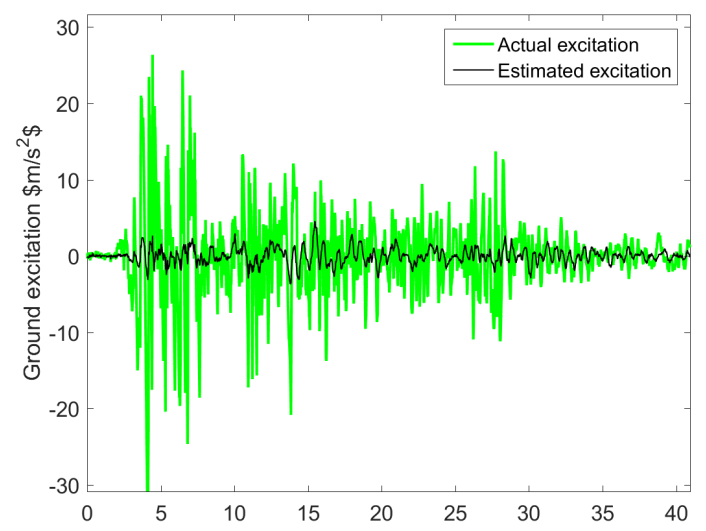

(d) 2 Channel measured signal

Figure 3: Estimation of input excitation for 16, 8, 4 and 2 channel measured signal 


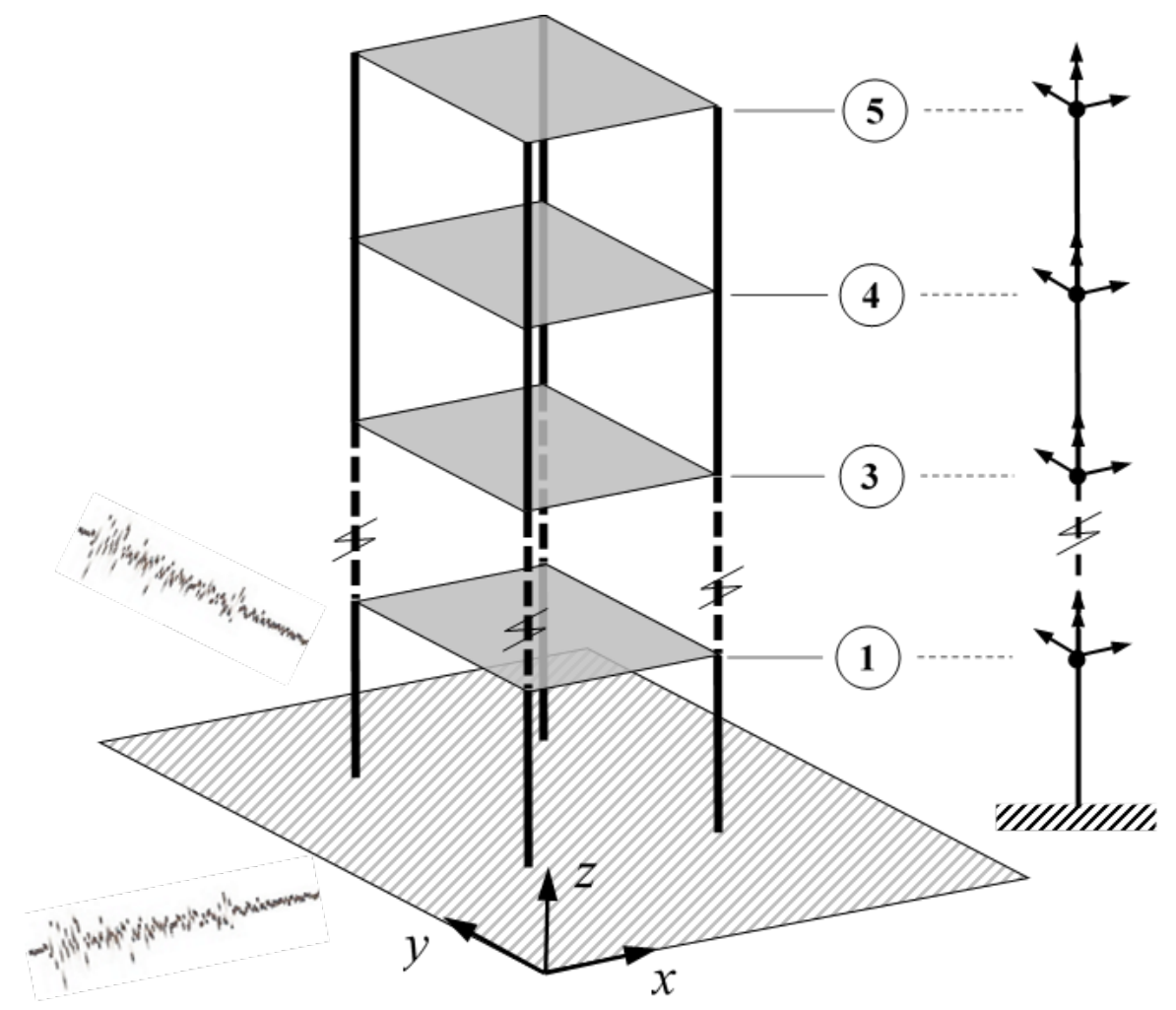

Figure 4: Schematic diagram of the five-story test structure

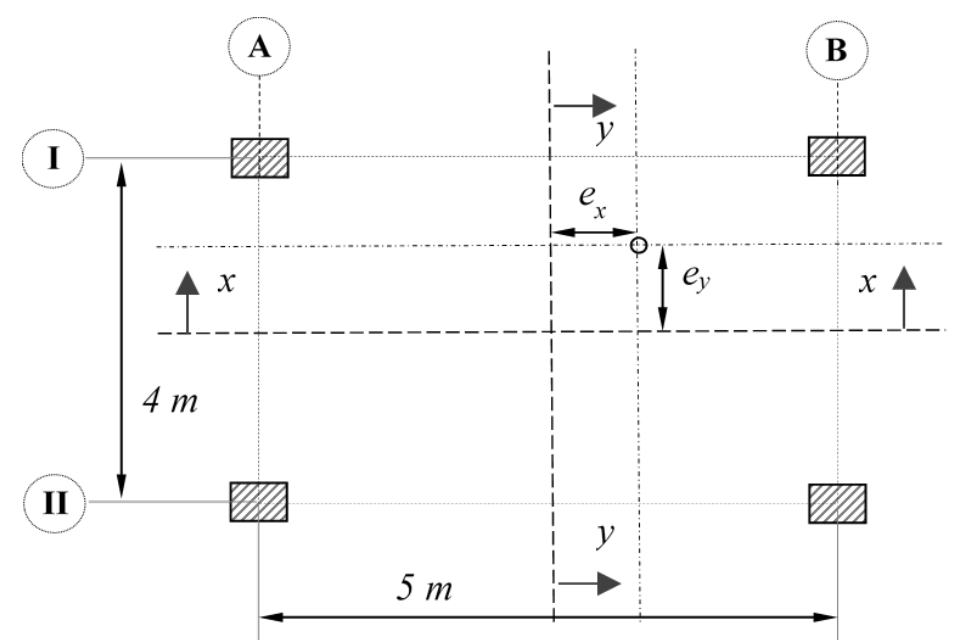

Figure 5: Plan of the five-story test structure 


\subsection{Five-story asymmetric building}

The second test structure is a five-story asymmetric building. The building is replicated using a three dimensional lumped mass model with each mass block having three free DOFs: two translational DOFs in direction $x$ and $y$ and a rotational DOF in $z$ direction. Figure 4 gives the schematic diagram of the test structure together with its lumped mass approximation. The column translational stiffnesses in direction $x$, $y$ and the rotational stiffness in direction $z$ are listed in Table 1. Based on the distribution (as described in Figure 5) of column stiffnesses, the center of rotation for the building is found to be offset by a distance of $0.4 m$ and $0.5 m$ in the direction of $x$ and $y$ respectively from the center. The mass blocks are assumed to have the same translational mass of $2,000 \mathrm{~kg}$ and rotational mass of $5,000 \mathrm{~kg}-\mathrm{m}^{2}$.

The simulation details for the lumped mass model of the test structure are given below. The local stiffness matrix for an arbitrary $i^{t h}$ story of the structure is constructed as:

$$
\mathbf{K}^{i}=\left[\begin{array}{cccccc}
k_{x_{i}} & 0 & -e_{y_{i}} k_{x_{i}} & -k_{x_{i}} & 0 & e_{y_{i}} k_{x_{i}} \\
0 & k_{y_{i}} & -e_{x_{i}} k_{y_{i}} & 0 & -k_{y_{i}} & e_{x_{i}} k_{y_{i}} \\
-e_{y_{i}} k_{x_{i}} & -e_{x_{i}} k_{y_{i}} & k_{x y_{i}} & e_{y_{i}} k_{x_{i}} & e_{x_{i}} k_{y_{i}} & -k_{x y_{i}} \\
-k_{x_{i}} & 0 & e_{y_{i}} k_{x_{i}} & k_{x_{i}} & 0 & -e_{y_{i}} k_{x_{i}} \\
0 & -k_{y_{i}} & e_{x_{i}} k_{y_{i}} & 0 & -k_{y_{i}} & -e_{x_{i}} k_{y_{i}} \\
e_{y_{i}} k_{x_{i}} & e_{x_{i}} k_{y_{i}} & -k_{x y_{i}} & -e_{y_{i}} k_{x_{i}} & -e_{x_{i}} k_{y_{i}} & k_{x y_{i}}
\end{array}\right]=\left[\begin{array}{cc}
K_{11}^{i} & K_{12}^{i} \\
K_{21}^{i} & K_{22}^{i}
\end{array}\right]
$$

where $k_{x}, k_{y}$ and $k_{x y}$ are the story stiffnesses in respective directions. $e_{x}$ and $e_{y}$ are the eccentricities of the rotation center of the building from the geometric center and have the value of $e_{x}=0.4 m$ and $e_{y}=0.5 m$. Using these local matrices, the unbounded global stiffness matrix is assembled as:

$$
\mathbf{K}=\left[\begin{array}{cccccc}
K_{11}^{1} & K_{12}^{1} & 0 & 0 & 0 & 0 \\
K_{21}^{1} & K_{22}^{1}+K_{11}^{2} & K_{12}^{2} & 0 & 0 & \\
0 & K_{21}^{2} & K_{22}^{2}+K_{11}^{3} & K_{12}^{3} & 0 & 0 \\
0 & 0 & K_{21}^{3} & K_{22}^{3}+K_{11}^{4} & K_{12}^{4} & 0 \\
0 & 0 & 0 & K_{21}^{4} & K_{22}^{4}+K_{11}^{5} & K_{12}^{5} \\
0 & 0 & 0 & 0 & K_{21}^{5} & K_{22}^{5}
\end{array}\right]
$$


The mass matrix is assembled as:

$$
\mathbf{M}=\left[\begin{array}{ccccc}
M^{1} & 0 & 0 & 0 & 0 \\
0 & M^{2} & 0 & 0 & 0 \\
0 & 0 & M^{3} & 0 & 0 \\
0 & 0 & 0 & M^{4} & 0 \\
0 & 0 & 0 & 0 & M^{5}
\end{array}\right] \text { with } \quad M^{i}=\left[\begin{array}{ccc}
M_{x} & 0 & 0 \\
0 & M_{y} & 0 \\
0 & 0 & I_{x y}
\end{array}\right]
$$

The damage in this building is incorporated by reducing all stiffness values at third floor level by $50 \%$. The damaged system is then base-excited using bi-directional El Centro earthquake excitation where its north-south and east-west components are applied in the $x$ and $y$ directions of the building respectively. The simulated response accelerations are measured at the sampling frequency of $50 \mathrm{~Hz}$ for a sample length of 2,048 which is then contaminated with $2 \%$ WGN as sensor noise. Three case studies are performed with i) measurements at all ten translational DOFs in $x$ and $y$ directions, ii) measurements at five translational DOFs (two in $x$ direction at the second and fourth floors and three in $y$ direction at the first, third and fifth floors) and ii) measurements at five translational DOFs (1,4,7,10 and 13) only in $x$ direction.

Table 1: Column stiffnesses in $x, y$ and rotational DOFs

\begin{tabular}{|l|l|l|l|}
\hline Column index & $k_{x} \mathrm{kN} / \mathrm{m}$ & $k_{y} \mathrm{kN} / \mathrm{m}$ & $k_{x y} \mathrm{kNm} / \mathrm{rad}$ \\
\hline A-I & 90 & 80 & 200 \\
\hline A-II & 60 & 80 & 200 \\
\hline B-I & 90 & 120 & 200 \\
\hline B-II & 60 & 120 & 200 \\
\hline
\end{tabular}

Once again, 2,000 particles are used for system identification and the same value for $\alpha$ and the initial value of $\mathbf{S}_{k \mid k}$ as in the previous example have been used. $1 \%$ of the base value of each stiffness component is considered as the $\sigma_{0}^{\xi}$.

The parameter estimation results are given in Figure 6a-6i for all three case studies. It is evident from the figures that with the decrease in the number of measurement signal channels, the estimation performance depletes (see Figure 6a-6f). It should also be noted that, while damage is occurring in both $x$ and $y$ directions, instrumenting the system only in $x$ direction leads to estimation of damage in $k_{x}$ only. The damage in $k_{y}$ is not sensed by the proposed algorithm (see Figure 6g-6i). This happens when there is weak correlation between stiffnesses in different directions, for which damage in one direction fails to show a prominent signature in response in other directions. Thus, damage in one direction is not observable from measurement in other directions. In this specific case, there is actually no correlation between $x$ and $y$ directions, which leads to a 


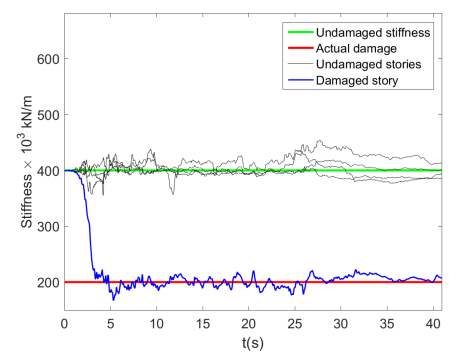

(a) Estimation of $k_{x}$

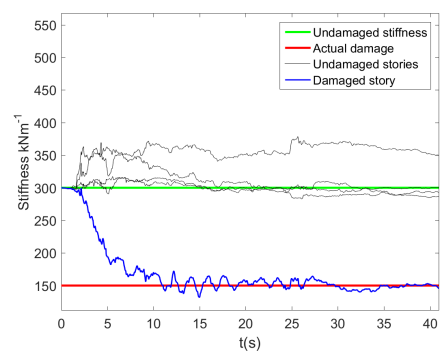

(b) Estimation of $k_{y}$

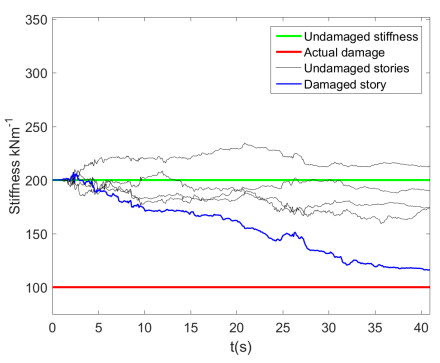

(c) Estimation of $k_{x y}$

Case Study 1: Ten channel measured signal: sensors in both directions ( $x$ and $y$ )

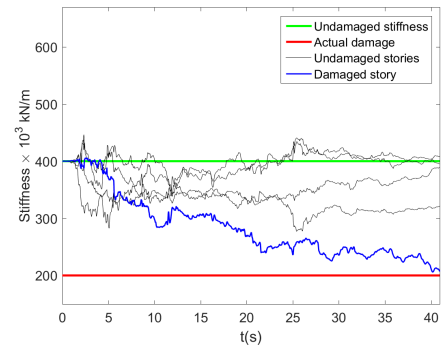

(d) Estimation of $k_{x}$

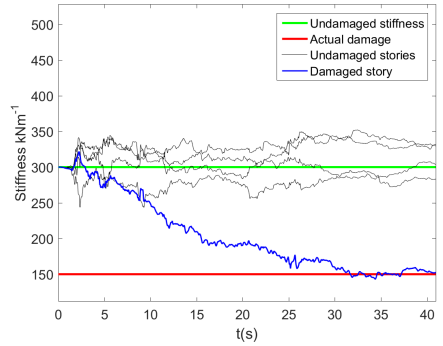

(e) Estimation of $k_{y}$

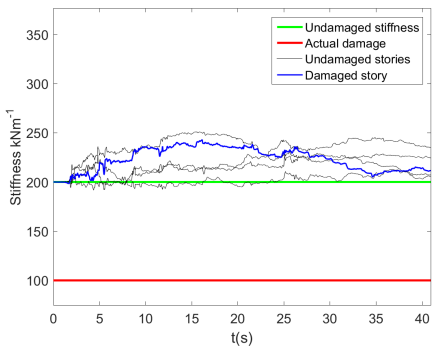

(f) Estimation of $k_{x y}$

Case Study 2: Five channel measured signal: sensors in both directions ( $x$ and $y$ )

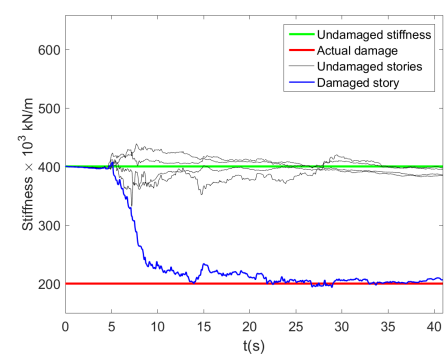

(g) Estimation of $k_{x}$

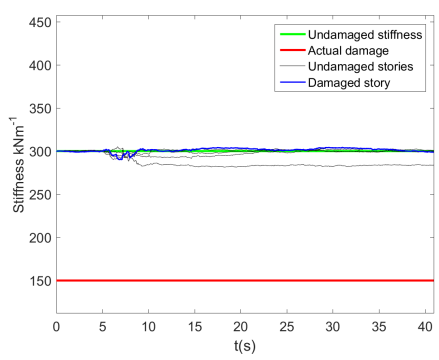

(h) Estimation of $k_{y}$

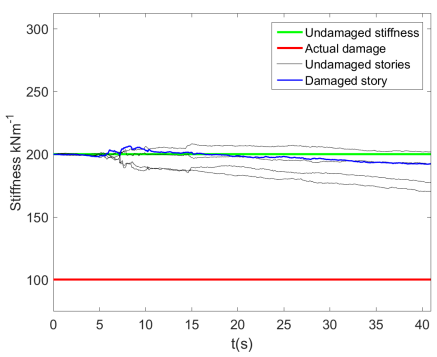

(i) Estimation of $k_{x y}$

Case Study 3: Five channel measured signal: sensors in only $x$ direction

Figure 6: Parameter estimation for all case studies. 
failure in detecting damage in $y$ direction. However, when the system is adequately instrumented (see Case Study 1), this weak correlation can still be sensed, which is evident from the results of the first case study.

From Figures 6a-6i it can be observed that, by reducing the number of measurement channels, the estimations are sometimes affected by unobservability issues. The reason behind this is the use of a simplistic model to define the system dynamics: each node is connected to its neighbouring nodes only by defining the correlation between nodal responses through a simple model. Response states at a particular node are therefore only observable if its immediate neighborhood is instrumented. Still, for real field SHM, simplistic system models are commonly employed in order to facilitate quick and cost-effective monitoring which obviously comes at the cost of accuracy. The results presented for this specific numerical simulation demonstrate that the damaged elements can be identified with acceptable accuracy.

The time varying selection of particle perturbation magnitude is further demonstrated in Figure 7. It can be observed that while the parameters corresponding to the damaged stories were changing, the perturbation width is also increased accordingly. On the other hand, for the undamaged stiffness entities, the perturbation width narrows down to its minimum specified level (i.e. $\delta \sigma_{0}^{\xi}$ ), allowing them to stabilize over time.

The estimated earthquake excitation for the first two successful case studies is presented in Figure 8. Again, a decrease in the precision of input estimation can be observed. Finally, a separate case study with ten measurement channels has been developed (with all other details the same as before) to demonstrate the robustness of the proposed algorithm. The system estimation is processed for a five-minute long collected measurement (time series of length chosen as 15,630 samples) before the earthquake arrived at the $100^{\text {th }}$ second causing damage initiation at the $105^{\text {th }}$ second. This example demonstrates that the proposed filter is stable over prolonged use and also sensitive to changes in the system whenever encountered. The results pertaining to this example are presented in Figures 9 and 10. Individual analysis of all three estimation results presented in Figure 9 might occasionally give a false perception of damage in undamaged stories. However, in order to identify the actually damaged story, one should analyze all three results simultaneously. Thus, if an index is set that takes a weighted sum of $k_{x}, k_{y}$ and $k_{x y}$, the actual damaged story can be easily identified without any ambiguity.

Finally, it should be noted that the measurements employed in these numerical experiments are base relative, which is not always available for real structures subjected to ground motion excitation. Special kinds of sensors (e.g. GPS antenna [41]) can be used to collect base relative measurements, but they are costly. The capability of this algorithm is, however, not limited to seismic force only, and can be extended 


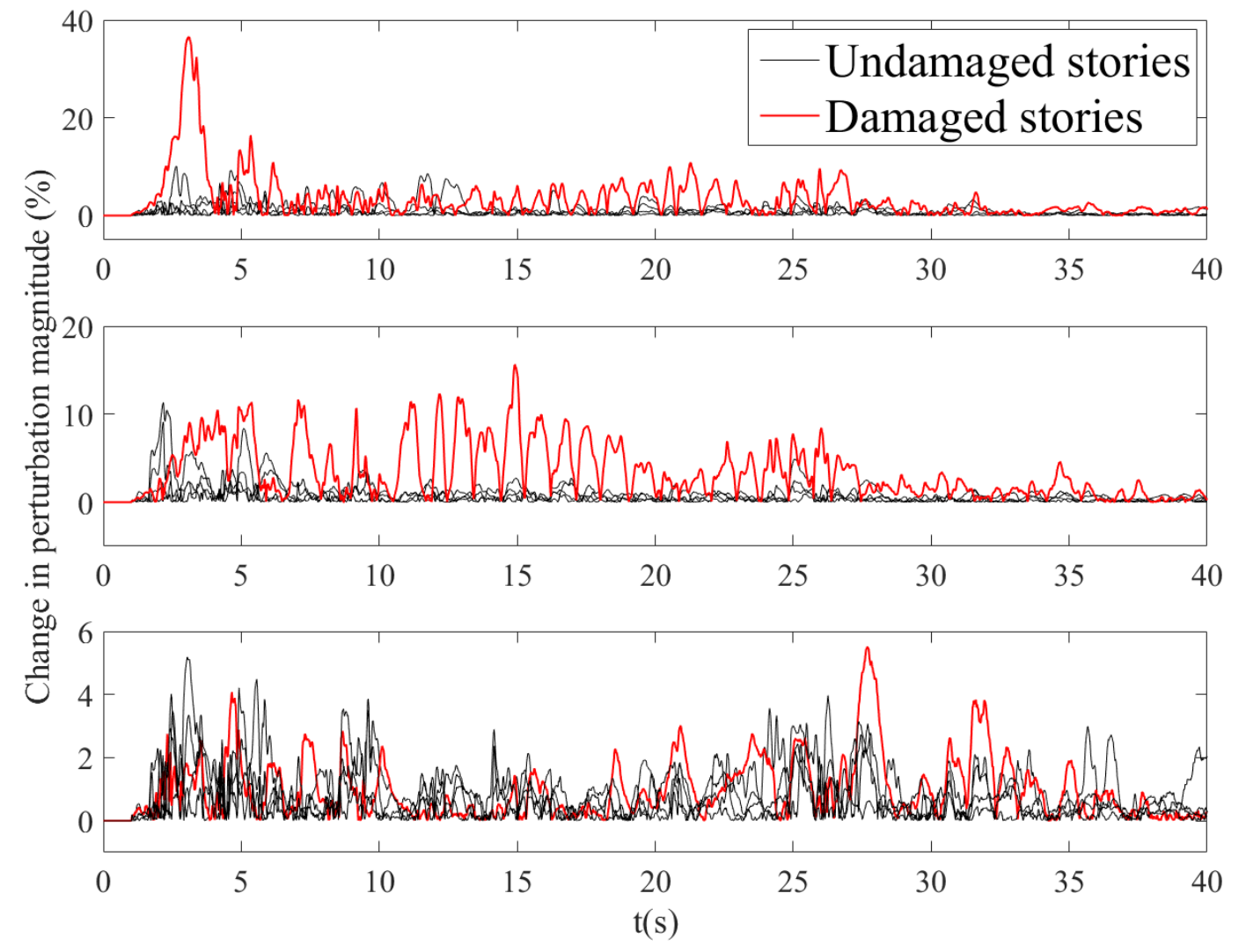

Figure 7: Variation of particle perturbation magnitude over time for $k_{x}, k_{y}$ and $k_{x y}$ : Case Study 1

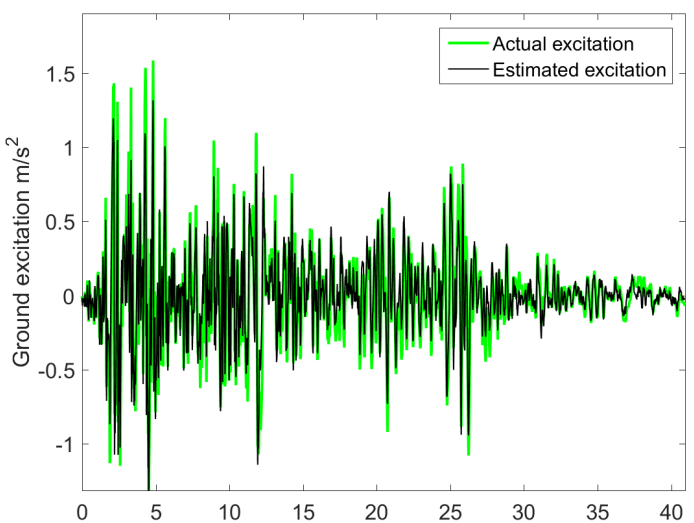

(a) Ten Channel measured signal

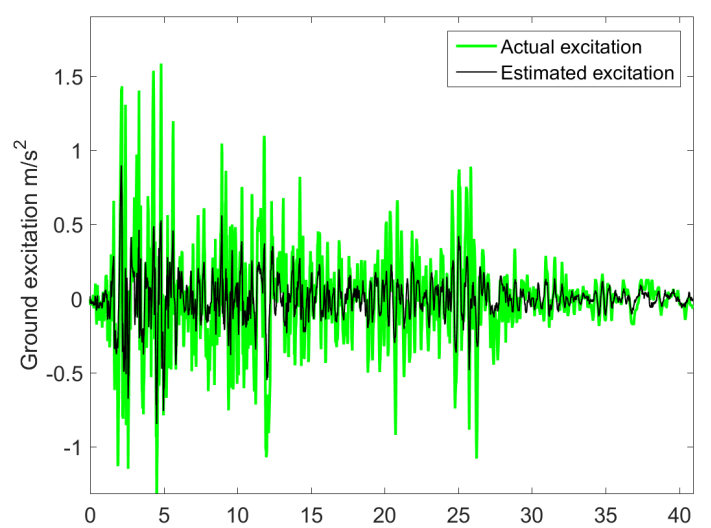

(b) Five Channel measured signal

Figure 8: Estimation of earthquake excitation on the building for ten and five channel measured signal 


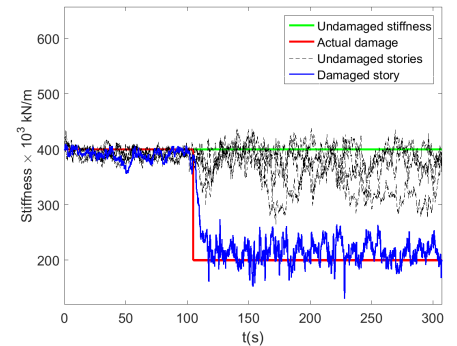

(a) Estimation of $k_{x}$

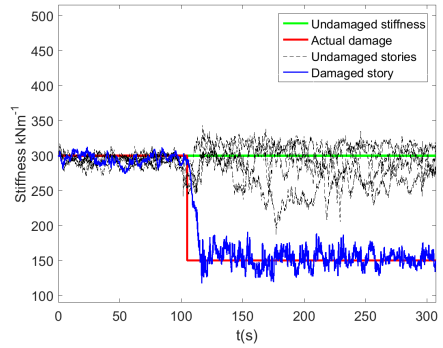

(b) Estimation of $k_{y}$

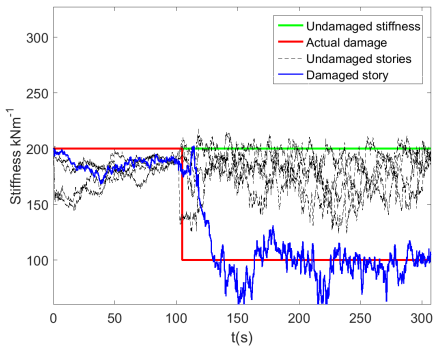

(c) Estimation of $k_{x y}$

Figure 9: simulation on robustness of the algorithm for ten-minute long system monitoring

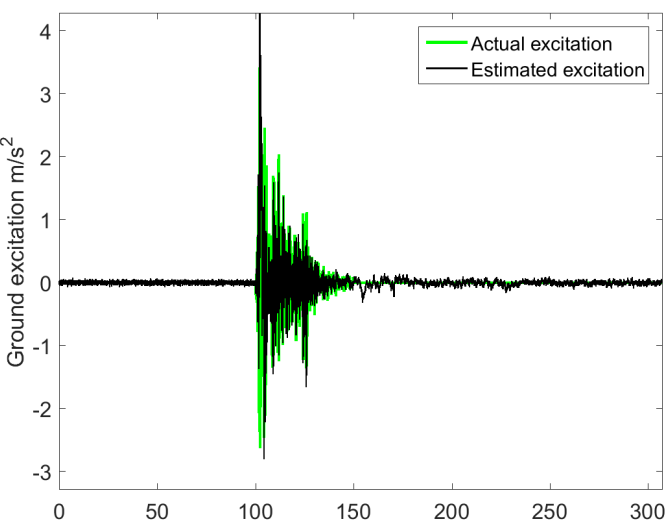

(a) Earthquake in $x$ direction

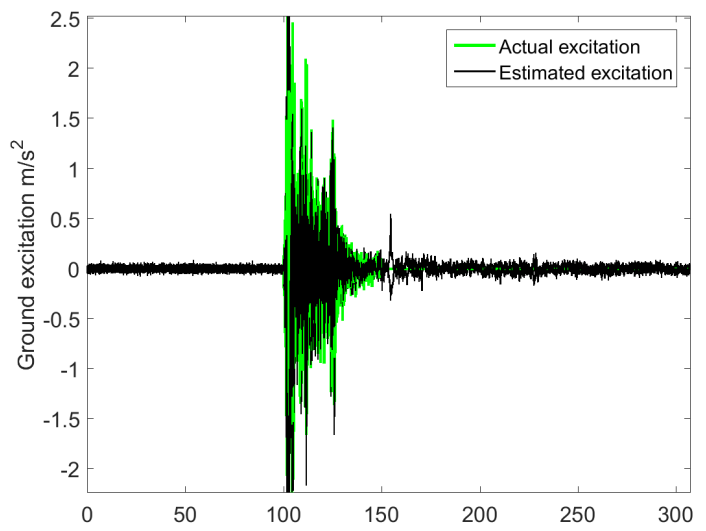

(b) Earthquake in $y$ direction

Figure 10: Earthquake excitation estimation for the robustness simulation 
for any force identification problems. For such problems, the measurement models always have to be changed depending on the available measurements. This proposal hopes to demonstrate the capability of the algorithm by means of a tractable numerical problem and therefore such a system definition has been adopted. Thus, in the absence of base-relative accelerations, the state vector should be observed through strain measurements (if available) using strain-displacement relationship as the measurement model. This will alleviate the requirement of base-relative measurements and make the algorithm more compatible for integration in real-world health monitoring systems. There exist numerical methods [52] for converting measured acceleration into inter-story drift ratio that can also be a cost effective measurement option for health estimation of real-life building infrastructures.

\section{Conclusion}

In this paper, we present a novel particle filter based technique to estimate the health parameters of systems subjected to an unknown input force of arbitrary distribution using its noise contaminated response measurement. This is done by incorporating a second filter parallel to the state-parameter estimation filter (i.e. IPKF) to identify the force and feed this information back to the first filter. The paper also defines a new gain matrix in order to handle systems with correlated process and measurement noises. A new particle evolution technique is also introduced that can help to stabilize the solution and thus indirectly alleviates the requirement of high number of particles to handle complex systems. Numerical simulations are performed on a mass-spring-damper system and a five-story building to demonstrate the proposed method successfully identifying the damage. A robustness test is also performed by running the proposed filter for a long time showing no instance of becoming unstable at any point in time.

\section{References}

[1] B. Peeters, G. De Roeck, Reference-based stochastic subspace identification for output-only modal analysis, Mechanical systems and signal processing 13 (6) (1999) 855-878.

[2] L. Mevel, A. Benveniste, M. Basseville, M. Goursat, B. Peeters, H. Van der Auweraer, A. Vecchio, Input/output versus output-only data processing for structural identificationapplication to in-flight data analysis, Journal of Sound and Vibration 295 (3) (2006) 531-552.

[3] G. Mercère, L. Bako, S. Lecœuche, Propagator-based methods for recursive subspace model identification, Signal Processing 88 (3) (2008) 468-491.

[4] I. Goethals, L. Mevel, A. Benveniste, B. De Moor, Recursive output-only subspace identification for in-flight flutter monitoring, in: Proceedings of the 22nd International Modal Analysis Conference (IMACXXII), Dearborn, Michigan, Vol. 7, 2004.

[5] G. Mercere, S. Lecoeuche, M. Lovera, Recursive subspace identification based on instrumental variable unconstrained quadratic optimization, International Journal of Adaptive Control and Signal Processing 18 (9-10) (2004) 771-797.

[6] S. Sen, B. Bhattacharya, A non-iterative structural damage identification methodology using state space eigenstructure assignment, Structure and Infrastructure Engineering 13 (2) (2017) 211-222. 
[7] S. Sen, B. Bhattacharya, Non-iterative eigenstructure assignment technique for finite element model updating, Journal of Civil Structural Health Monitoring 5 (4) (2015) 365-375.

[8] M. Hoshiya, E. Saito, Structural identification by extended kalman filter, Journal of Engineering Mechanics 110 (12) (1984) 1757-1770.

[9] R. Ghanem, M. Shinozuka, Structural-system identification. i: Theory, Journal of Engineering Mechanics 121 (2) (1995) 255-264.

[10] O. Maruyama, M. Hoshiya, System identification of an experimental model by extended kalman filter., Structural Safety and Reliability: ICOSSAR'01 (2001) 2001.

[11] B. Radhika, C. Manohar, Nonlinear dynamic state estimation in instrumented structures with conditionally linear gaussian substructures, Probabilistic Engineering Mechanics 30 (2012) 89-103.

[12] B. Radhika, C. Manohar, Updating response sensitivity models of nonlinear vibrating structures using particle filters, Computers \& Structures 89 (11) (2011) 901-911.

[13] H. Nasrellah, C. Manohar, Particle filters for structural system identification using multiple test and sensor data: a combined computational and experimental study, Structural Control and Health Monitoring 18 (1) (2011) 99-120.

[14] S. J. Julier, J. K. Uhlmann, New extension of the kalman filter to nonlinear systems, in: AeroSense'97, International Society for Optics and Photonics, 1997, pp. 182-193.

[15] S. Mariani, A. Ghisi, Unscented kalman filtering for nonlinear structural dynamics, Nonlinear Dynamics 49 (1) (2007) 131150.

[16] J. Ching, J. L. Beck, K. A. Porter, Bayesian state and parameter estimation of uncertain dynamical systems, Probabilistic engineering mechanics 21 (1) (2006) 81-96.

[17] E. N. Chatzi, A. W. Smyth, The unscented kalman filter and particle filter methods for nonlinear structural system identification with non-collocated heterogeneous sensing, Structural control and health monitoring 16 (1) (2009) 99-123.

[18] S. E. Azam, M. Bagherinia, S. Mariani, Stochastic system identification via particle and sigma-point kalman filtering, Scientia Iranica 19 (4) (2012) 982-991.

[19] C. Snyder, T. Bengtsson, P. Bickel, J. Anderson, Obstacles to high-dimensional particle filtering, Monthly Weather Review 136 (12) (2008) 4629-4640.

[20] A. Al-Hussein, A. Haldar, Novel unscented kalman filter for health assessment of structural systems with unknown input, Journal of Engineering Mechanics 141 (7) (2015) 04015012.

[21] A. Al-Hussein, A. Haldar, Unscented kalman filter with unknown input and weighted global iteration for health assessment of large structural systems, Structural Control and Health Monitoring 23 (1) (2016) 156-175.

[22] A. Corigliano, S. Mariani, Parameter identification in explicit structural dynamics: performance of the extended kalman filter, Computer Methods in Applied Mechanics and Engineering 193 (36) (2004) 3807-3835.

[23] A. Doucet, N. De Freitas, K. Murphy, S. Russell, Rao-blackwellised particle filtering for dynamic bayesian networks, in: Proceedings of the Sixteenth conference on Uncertainty in artificial intelligence, Morgan Kaufmann Publishers Inc., 2000, pp. 176-183.

[24] P. Li, R. Goodall, V. Kadirkamanathan, Estimation of parameters in a linear state space model using a rao-blackwellised particle filter, IEE Proceedings-control theory and applications 151 (6) (2004) 727-738.

[25] P. Del Moral, F.-K. Formulae, genealogical and interacting particle systems with applications, probability and its applications (2004).

[26] Z. Chen, et al., Bayesian filtering: From kalman filters to particle filters, and beyond, Statistics 182 (1) (2003) 1-69.

[27] G. Capellari, S. Eftekhar Azam, S. Mariani, Damage detection in flexible plates through reduced-order modeling and hybrid particle-kalman filtering, Sensors 16 (1) (2015) 2.

[28] S. Sen, B. Bhattacharya, Progressive damage identification using dual extended kalman filter, Acta Mechanica 227 (8) (2016) 2099-2109.

[29] S. Sen, B. Bhattacharya, Online structural damage identification technique using constrained dual extended kalman filter, Structural Control and Health Monitoring.

[30] S. E. Azam, A. Ghisi, S. Mariani, Parallelized sigma-point kalman filtering for structural dynamics, Computers \& Structures 92 (2012) 193-205.

[31] S. E. Azam, E. Chatzi, C. Papadimitriou, A dual kalman filter approach for state estimation via output-only acceleration measurements, Mechanical Systems and Signal Processing 60 (2015) 866-886.

[32] L. Ljung, Asymptotic behavior of the extended kalman filter as a parameter estimator for linear systems, IEEE Transactions on Automatic Control 24 (1) (1979) 36-50.

[33] M. Zghal, L. Mevel, P. Del Moral, Modal parameter estimation using interacting kalman filter, Mechanical Systems and Signal Processing 47 (1) (2014) 139-150.

[34] A. Crinière, M. Zghal, L. Mevel, J. Dumoulin, Gpgpu implementation of modal meter tracking by particle based kalman filter, in: 8th European Workshop On Structural Health Monitoring (EWSHM 2016), 2016. 
[35] P. K. Kitanidis, Unbiased minimum-variance linear state estimation, Automatica 23 (6) (1987) 775-778.

[36] C.-S. Hsieh, Robust two-stage kalman filters for systems with unknown inputs, IEEE Transactions on Automatic Control 45 (12) (2000) 2374-2378.

[37] S. Gillijns, B. De Moor, Unbiased minimum-variance input and state estimation for linear discrete-time systems, Automatica 43 (1) (2007) 111-116.

[38] S. Gillijns, B. De Moor, Unbiased minimum-variance input and state estimation for linear discrete-time systems with direct feedthrough, Automatica 43 (5) (2007) 934-937.

[39] E. Lourens, C. Papadimitriou, S. Gillijns, E. Reynders, G. De Roeck, G. Lombaert, Joint input-response estimation for structural systems based on reduced-order models and vibration data from a limited number of sensors, Mechanical Systems and Signal Processing 29 (2012) 310-327.

[40] E. Lourens, E. Reynders, G. De Roeck, G. Degrande, G. Lombaert, An augmented kalman filter for force identification in structural dynamics, Mechanical Systems and Signal Processing 27 (2012) 446-460.

[41] R. Astroza, H. Ebrahimian, Y. Li, J. P. Conte, Bayesian nonlinear structural fe model and seismic input identification for damage assessment of civil structures, Mechanical Systems and Signal Processing 93 (2017) 661-687.

[42] F. Naets, J. Croes, W. Desmet, An online coupled state/input/parameter estimation approach for structural dynamics, Computer Methods in Applied Mechanics and Engineering 283 (2015) 1167-1188.

[43] S. Eftekhar Azam, V. K. Dertimanis, E. Chatzi, C. Papadimitriou, Output-only schemes for joint input-state-parameter estimation of linear systems, in: Proceedings of the 1st International Conference on Uncertainty Quantification in Computational Sciences and Engineering (UNCECOMP 2015), National Technical University of Athens, 2015, pp. 497-510.

[44] E. Reynders, G. De Roeck, Reference-based combined deterministic-stochastic subspace identification for experimental and operational modal analysis, Mechanical Systems and Signal Processing 22 (3) (2008) 617-637.

[45] P. Van Overschee, B. De Moor, Subspace identification for linear systemskluwer academic publishers, ISBN-13 (1996) 9780792397175

[46] K. Maes, A. Smyth, G. De Roeck, G. Lombaert, Joint input-state estimation in structural dynamics, Mechanical Systems and Signal Processing 70 (2016) 445-466.

[47] G. Bishop, G. Welch, An introduction to the kalman filter, Proc of SIGGRAPH, Course 8 (27599-23175) (2001) 41.

[48] J. Liu, M. West, Combined parameter and state estimation in simulation-based filtering, in: Sequential Monte Carlo methods in practice, Springer, 2001, pp. 197-223.

[49] M. West, Approximating posterior distributions by mixture, Journal of the Royal Statistical Society. Series B (Methodological) (1993) 409-422.

[50] M. West, Mixture models, monte carlo, bayesian updating, and dynamic models, Computing Science and Statistics (1993) $325-325$

[51] F. Gustafsson, F. Gunnarsson, N. Bergman, U. Forssell, J. Jansson, R. Karlsson, P.-J. Nordlund, Particle filters for positioning, navigation, and tracking, IEEE Transactions on signal processing 50 (2) (2002) 425-437.

[52] D. A. Skolnik, J. W. Wallace, Critical assessment of interstory drift measurements, Journal of structural engineering 136 (12) (2010) 1574-1584

\section{Appendix A. Modification in gain calculation}

In this section, the derivation of the improved gain matrix is discussed for the system described in

Equation 3. We start with the prediction of state mean and covariance as follows:

$$
\begin{aligned}
\mathbf{x}_{k \mid k-1}^{i}=E\left[\mathbf{x}_{k}^{i} \mid \mathbf{y}_{1: k-1}\right] & =\mathbf{F}_{k}^{i} \mathbf{x}_{k-1 \mid k-1}^{i} \\
\mathbf{P}_{k \mid k-1}^{i}=\operatorname{COV}\left[\mathbf{x}_{k}^{i} \mid \mathbf{y}_{1: k-1}\right] & =\mathbf{F}_{k}^{i} \mathbf{P}_{k-1 \mid k-1}^{i} \mathbf{F}_{k}^{i T}+\mathbf{B S}_{k-1 \mid k-1} \mathbf{B}^{T}+\mathbf{G Q G}^{T}
\end{aligned}
$$


$\mathbf{S}_{k-1 \mid k-1}$ has been employed as the best estimate for $\operatorname{COV}\left[\mathbf{u}_{k} \mid \mathbf{y}_{1: k-1}\right]$. The other covariance terms are estimated as:

$$
\begin{aligned}
& \operatorname{COV}\left[\mathbf{x}_{k}^{i} ; \mathbf{y}_{k} \mid \mathbf{y}_{1: k-1}\right]=\operatorname{COV}\left[\left\{\mathbf{F}_{k}^{i} \mathbf{x}_{k-1}^{i}+\mathbf{B} \mathbf{u}_{k}+\mathbf{G v}_{k}\right\}\right. \\
& \left.\quad\left\{\mathbf{H}_{k}^{i}\left(\mathbf{F}_{k}^{i} \mathbf{x}_{k-1}^{i}+\mathbf{B} \mathbf{u}_{k}+\mathbf{G v}_{k}\right)+\mathbf{D} \mathbf{u}_{k}+\mathbf{L} \mathbf{v}_{k}+\mathbf{w}_{k}\right\} \mid \mathbf{y}_{1: k-1}\right] \\
& =\mathbf{F}_{k}^{i} \mathbf{P}_{k-1 \mid k-1}^{i} \mathbf{F}_{k}^{i T} \mathbf{H}_{k}^{i T}+\mathbf{B S}_{k-1 \mid k-1} \mathbf{B}^{T} \mathbf{H}_{k}^{i T}+\mathbf{B S}_{k-1 \mid k-1} \mathbf{D}^{T}+\mathbf{G Q G}^{T} \mathbf{H}_{k}^{i T}+\mathbf{G Q L}^{T}
\end{aligned}
$$

and likewise,

$$
\begin{aligned}
& \operatorname{COV}\left[\mathbf{y}_{k} ; \mathbf{y}_{k} \mid \mathbf{y}_{1: k-1}\right]=\mathbf{H}_{k}^{i} \mathbf{P}_{k \mid k-1}^{i} \mathbf{H}_{k}^{i T}+\mathbf{H}_{k}^{i} \mathbf{B S}_{k-1 \mid k-1} \mathbf{D}^{T}+\mathbf{D S}_{k-1 \mid k-1} \mathbf{B}^{T} \mathbf{H}_{k}^{i}+\mathbf{D S}_{k-1 \mid k-1} \mathbf{D}^{T} \\
& +\mathbf{H}_{k}^{i} \mathbf{G Q L}^{T}+\mathbf{L Q G}^{T} \mathbf{H}_{k}^{i}+\mathbf{L Q L}^{T}+\mathbf{R}
\end{aligned}
$$

The gain can then be estimated as:

$$
\mathbf{K}_{k}^{i}=\operatorname{COV}\left[\mathbf{x}_{k}^{i} ; \mathbf{y}_{k} \mid \mathbf{y}_{1: k-1}\right] \operatorname{COV}\left[\mathbf{y}_{k} ; \mathbf{y}_{k} \mid \mathbf{y}_{1: k-1}\right]^{-1}
$$

